\title{
The p-Laplacian equation in thin domains: The unfolding approach
}

\author{
José M. Arrieta*1 $^{*}$, Jean Carlos Nakasato ${ }^{\dagger 2}$ and Marcone Corrêa Pereira ${ }^{\ddagger 2}$ \\ ${ }^{1}$ Depto. de Análisis Matemático y Matemática Aplicada, Universidad Complutense de \\ Madrid, 28040 Madrid, Spain \\ ${ }^{2}$ Depto. Matemática Aplicada, IME, Universidade de São Paulo, Rua do Matão 1010, São \\ Paulo - SP, Brazil
}

2018

\begin{abstract}
In this work we apply the unfolding operator method to analyze the asymptotic behavior of the solutions of the $p$-Laplacian equation with Neumann boundary condition set in a bounded thin domain of the type $R^{\varepsilon}=\left\{(x, y) \in \mathbb{R}^{2}: x \in(0,1)\right.$ and $\left.0<y<\varepsilon g\left(x / \varepsilon^{\alpha}\right)\right\}$ where $g$ is a positive periodic function. We study the three cases $0<\alpha<1, \alpha=1$ and $\alpha>1$ representing respectively weak, resonant and high osillations at the top boundary. In the three cases we deduce the homogenized limit and obtain correctors.
\end{abstract}

Keywords: p-Laplacian, Neumann boundary condition, Thin domains, Oscillatory boundary, Homogenization.

2010 Mathematics Subject Classification. 35B25, 35B40, 35J92.

\section{Introduction}

Let $R^{\varepsilon} \subset \mathbb{R}^{2}$ be the following family of thin domains

$$
R^{\varepsilon}=\left\{(x, y) \in \mathbb{R}^{2}: x \in(0,1) \text { and } 0<y<\varepsilon g\left(\frac{x}{\varepsilon^{\alpha}}\right)\right\}, \quad \varepsilon>0,
$$

where $\alpha>0$ is a fixed parameter, $g: \mathbb{R} \rightarrow \mathbb{R}$ is a strictly positive function, periodic of period $L$, lower semicontinuous satisfying

$$
0<g_{0} \leq g(x) \leq g_{1}, \quad \forall x \in(0, L)
$$

with $g_{0}=\min _{x \in \mathbb{R}} g(x)$ and $g_{1}=\sup _{x \in \mathbb{R}} g(x)$.

In this work, we are interested in analyzing the asymptotic behavior of the family of solutions of the nonlinear elliptic equation

$$
\left\{\begin{array}{l}
-\Delta_{p} u_{\varepsilon}+\left|u_{\varepsilon}\right|^{p-2} u_{\varepsilon}=f^{\varepsilon} \text { in } R^{\varepsilon} \\
\left|\nabla u_{\varepsilon}\right|^{p-2} \nabla u_{\varepsilon} \eta_{\varepsilon}=0 \text { on } \partial R^{\varepsilon}
\end{array}\right.
$$

where $\eta_{\varepsilon}$ is the unit outward normal vector to the boundary $\partial R^{\varepsilon}, 1<p<\infty$ and

$$
\Delta_{p} \cdot=\operatorname{div}\left(|\nabla \cdot|^{p-2} \nabla \cdot\right)
$$

denotes the $p$-Laplacian differential operator. We also assume $f^{\varepsilon} \in L^{p^{\prime}}\left(R^{\varepsilon}\right)$ where $p^{\prime}$ is the conjugate exponent of $p$, that is $1 / p^{\prime}+1 / p=1$.

*e-mail: arrieta@mat.ucm.es.

†e-mail: nakasato@ime.usp.br.

‡e-mail: marcone@ime.usp.br. 
It is known that the variational formulation of $(2)$ is given by

$$
\int_{R^{\varepsilon}}\left\{\left|\nabla u_{\varepsilon}\right|^{p-2} \nabla u_{\varepsilon} \nabla \varphi+\left|u_{\varepsilon}\right|^{p-2} u_{\varepsilon} \varphi\right\} d x d y=\int_{R^{\varepsilon}} f^{\varepsilon} \varphi d x d y, \quad \varphi \in W^{1, p}\left(R^{\varepsilon}\right) .
$$

Furthermore, for each fixed $\varepsilon>0$ the existence and uniqueness of solutions is guaranteed by MintyBrowder's Theorem. Hence, we are interested here in analyzing the asymptotic behavior of the solutions $u_{\varepsilon}$ as $\varepsilon \rightarrow 0$, that is, as the domain $R^{\varepsilon}$ becomes thinner and thinner although with a high oscillating boundary at the top.

Indeed, since the set $R^{\varepsilon} \subset(0,1) \times\left(0, \varepsilon g_{1}\right)$ for all $\varepsilon>0$, we have the parameter $\varepsilon$ models the thin domain situation. Moreover, we see that $R^{\varepsilon}$ has tickness of order $\varepsilon$, and then, it is expected that for $\varepsilon \approx 0$ the sequence of solutions $u_{\varepsilon}$ will converge to a function of just one single variable $x \in(0,1)$ and that this function will satisfy an equation of the same type as $(2)$ but in one dimension.

On the other side, the parameter $\alpha$ measures the intensity of the oscillations of the top boundary and, as we will see, the homogenized limit equation will depend tightly on this positive number. We will deal with three distinct cases: weak oscillatory case $(0<\alpha<1)$, the resonant or critical case $(\alpha=1)$, and the high oscillatory one $(\alpha>1)$ See Figure 1 below where these three cases are illustrated. We will obtain different limit problems according to this three cases.

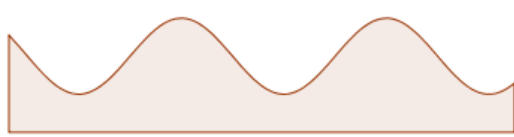

(a) Weak oscillation: $\alpha<1$.

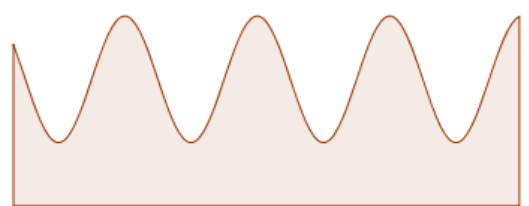

(b) Resonant case: $\alpha=1$.

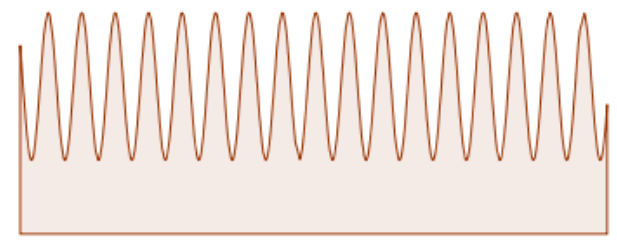

(c) High oscillation: $\alpha>1$.

Figure 1: Examples of oscillatory behavior at the boundary $\partial R^{\varepsilon}$.

Here we will combine techniques such as unfolding operator methods for thin domains, which were developed in [5, 6], as well as, those ones presented in [9, 10] in order to analyze monotone operators in perforated domains. Furthermore, we will also obtain corrector results for each case considered here.

As we will see in this work, the homogenized limit problem is given by the following one-dimensional $p$-Laplacian equation with constant coefficient $q$ :

$$
\left\{\begin{array}{c}
-q\left(\left|u^{\prime}\right|^{p-2} u^{\prime}\right)^{\prime}+|u|^{p-2} u=\bar{f} \quad \text { in }(0,1) \\
u^{\prime}(0)=u^{\prime}(1)=0 .
\end{array} .\right.
$$

Indeed, the coefficient $q$ has different expresion for the three different cases of $\alpha$. As a matter of fact, for $\alpha=1$ in (1), we show that the homogenized coefficient $q$ is a positive constant and it is given by

$$
q=\frac{1}{\left|Y^{*}\right|} \int_{Y^{*}}|\nabla v|^{p-2} \partial_{y_{1}} v d y_{1} d y_{2}
$$

where $Y^{*}$ is the representative cell of the oscillating domain $R^{\varepsilon}$

$$
Y^{*}=\left\{\left(y_{1}, y_{2}\right) \in \mathbb{R}^{2}: 0<y_{1}<L \text { and } 0<y_{2}<g\left(y_{1}\right)\right\}
$$


The function $v$ appearing in (5) is an auxiliar function, which is the unique solution of the following problem

$$
\begin{array}{cl}
\int_{Y^{*}}|\nabla v|^{p-2} \nabla v \nabla \varphi d y_{1} d y_{2}=0 & \forall \varphi \in W_{\#}^{1, p}\left(Y^{*}\right), \\
\left(v-y_{1}\right) \in W_{\#}^{1, p}\left(Y^{*}\right) \quad \text { with } & \left\langle\left(v-y_{1}\right)\right\rangle_{Y^{*}}=0,
\end{array}
$$

where

$$
W_{\#}^{1, p}\left(Y^{*}\right)=\left\{\varphi \in W^{1, p}\left(Y^{*}\right):\left.\varphi\right|_{\partial_{l e f t} Y^{*}}=\left.\varphi\right|_{\partial_{\text {right }} Y^{*}}\right\}
$$

is the space of periodic functions on the horizontal variable $x$, and $\langle\varphi\rangle_{\mathcal{O}}$ denotes the average of the function $\varphi \in L_{\text {loc }}^{1}\left(\mathbb{R}^{N}\right)$ on the open set $\mathcal{O} \subset \mathbb{R}^{N}$.

It is worth noting that problem (7) is well posed due again to Minty-Browder's Theorem. This implies that $q$ is well defined and is a positive constant (see (34)). Moreover, we have that the forcing term $\bar{f}$ of the limit equation (4) is obtained as the limit of the unfolding operator acting on functions $f^{\varepsilon}$ (see for instance (12) and (14) below).

For the case $\alpha<1$, we obtain that the homogenized coefficient depends just on the function $g$, which describes the profile of the oscillatory boundary and on the number $p \in(1, \infty)$, which establishes the order of the $p$-Laplacian operator. It gets the following form

$$
q=\frac{1}{\langle g\rangle_{(0, L)}\left\langle 1 / g^{p^{\prime}-1}\right\rangle_{(0, L)}^{p-1}} .
$$

We still mention that the forcing term $\bar{f}$ is also given by $(12)$ and 14 since it is computed in the same way that in the previous case $\alpha=1$.

Finally, for the case $\alpha>1$, we first note that forcing term $\bar{f}$ gets a different expression. Since it is computed in a different way, not anymore as a consequence of the unfolding operator, it takes the form (42). The homogenized coefficient $q$ of the limit equation (4) now assumes the form

$$
q=\frac{g_{0}}{\langle g\rangle_{(0, L)}}
$$

It does not depend explicitly on $p$, but on $g_{0}$, the minimum value of the $L$-periodic function $g$, which is strictly positive. For this case, it is easy to see that $q<1$ if $g$ is not constant. In fact,

$$
\frac{g_{0}}{\langle g\rangle_{(0, L)}}=\frac{L g_{0}}{\left|Y^{*}\right|}<\frac{\left|Y^{*}\right|}{\left|Y^{*}\right|}=1
$$

Somehow, we can say that the high oscillatory behavior tends to affect the system in such way that its diffusion becomes smaller. Notice that $q$ also has a lower bound in the class of functions $g$ considered here. It satisfies $q \geq g_{0} / g_{1}$ where $g_{1}$ is the maximum value of $g$ in $[0, L]$.

Complete statements on the homogenized limit problems and the corresponding convergence of solutions are stated in Theorem 3.1 for $\alpha=1$, Theorem 4.1 for $0<\alpha<1$, and Theorem 5.3 for $\alpha>1$. Strong convergence in Sobolev spaces like $W^{1, p}$ are also obtained using the corrector approach discussed for instance in [6, 9]. We show the existence of a family of functions $W_{\varepsilon}$ such that

$$
\varepsilon^{-1}\left\|\nabla u_{\varepsilon}-W_{\varepsilon}\right\|_{L^{p}\left(R^{\varepsilon}\right)}^{p} \rightarrow 0, \quad \text { as } \varepsilon \rightarrow 0 .
$$

Such results are precisely stated in Corollary 3.1.1, 4.1.1 and 5.3.1, respectively for each case: $\alpha=1$, $0<\alpha<1$ and $\alpha>1$.

Now, let us notice that there are several works in the literature dealing with issues related to the effect of thickness and roughness on the feature of the solutions of partial differential equations. Indeed, thin structures with oscillating boundaries appear in many fields of science: fluid dynamics (lubrication), solid mechanics (thin rods, plates or shells) or even physiology (blood circulation). Therefore, analyzing the asymptotic behavior of different models on thin structures and understanding how the geometry and the roughness affects the limit problem is a very relevant issues in applied science. Here, we just mention some works in these directions [1, 17, 8, 11, 12, 15, 17, 18]. 
Furthermore, we point out that the particular case taking $p=2$ in equation (2), which represents the Laplace differential operator, has been originally discussed in some previous works using different techniques and methods. Indeed, in [20] the author among other things, treats the case of $0<\alpha<1$ (even with a doubly oscillatory boundary) via change of variables and rescaling the thin domain as in the classical work [13]. The resonant case, $\alpha=1$, has been studied in [2, 3, 15] where techniques from homogenization theory have been used.

The case with fast oscillatory boundary $(\alpha>1)$ was obtained in 4 by decomposing the domain in two parts separating the oscillatory boundary. There, the authors also consider more general and complicated geometries which are not given as the graph of certain smooth functions. See also [6, 20].

In [5, 6], the authors introduce the unfolding method in thin domains tackling these three cases for the Laplace operator with Neumann boundary condition in a unified way. Also, the regularity requirement on the function $g$ is very mild.

Now, concerning with the $p$-Laplacian, we have recently applied techniques from [2, 9, 10] to obtain the limit equation and corrector results in smooth thin domains for the resonant case in [16] improving previous works such as [19].

The main goal of this paper is to improve the previous mentioned works considering the p-Laplacian equation. As a matter of fact, combining techniques from [9, 10] and [5, 6], we will be able to deal with equation (2) on non-smooth oscillating thin domains for any $1<p<\infty$ and any order of oscillation $\alpha>0$.

Notice that this is not a easy task since we are studying here a quasilinear differential equation, which can be singular, as it is in the case $1<p<2$, or degenerated, if $2<p<\infty$. Moreover, the problem is posed in non-smooth thin domains like comb-like thin domains where standard extension operators do not apply (see figure 2). Besides, it is worth observing that the unfolding method also allows us to obtain some new strong convergence results for the solutions by corrector approach.

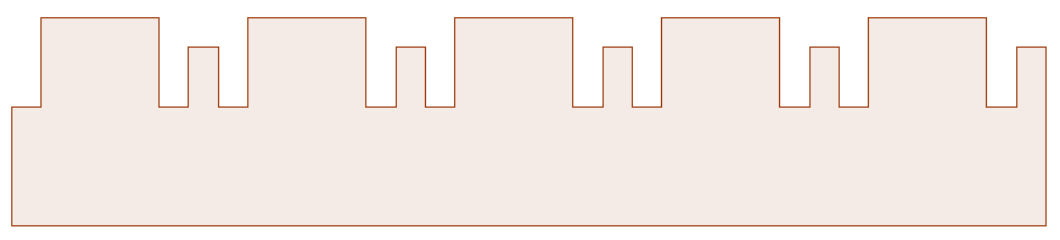

Figure 2: Comb-like thin domains.

The paper is organized as follows: in Section 2, we state some notations and basic results. In Section 3, we consider the resonant case $\alpha=1$ obtaining the homogenized equation via a somehow classical auxiliar problem given by homogenization theory. In Section 4 , the weak oscillation case is studied, and in Section 5, we finally consider the case of thin domains with very highly oscillatory boundaries.

\section{$2 \quad$ Notations and Basic Facts}

To study the convergence of the solutions of (3), we clarify some notation and recall some results concerning monotone operators and the method of unfolding operator. We will need these results for our analysis.

We consider two-dimensional thin domains defined by (1). Observe that this domains have an oscillatory behavior at its top boundary. The parameters $\varepsilon$ and $\alpha$ are positive and the function $g$ satisfies the following hypothesis

$\left(\mathbf{H}_{\mathbf{g}}\right) g: \mathbb{R} \rightarrow \mathbb{R}$ is a strictly positive, bounded, lowersemicontinuous, L-periodic function. Moreover, we define

$$
g_{0}=\min _{x \in \mathbb{R}} g(x) \quad \text { and } \quad g_{1}=\max _{x \in \mathbb{R}} g(x)
$$

so that $0<g_{0} \leq g(x) \leq g_{1}$ for all $x \in \mathbb{R}$

Recall that lower semicontinuous means that $g\left(x_{0}\right) \leq \liminf _{x \rightarrow x_{0}} g(x), \forall x_{0} \in \mathbb{R}$. 
Recall that $Y^{*}$, given by (6) is the basic cell of the thin domain $R^{\varepsilon}$ and

$$
\langle\varphi\rangle_{\mathcal{O}}:=\frac{1}{|\mathcal{O}|} \int_{\mathcal{O}} \varphi(x) d x
$$

is the average of $\varphi \in L_{l o c}^{1}\left(\mathbb{R}^{2}\right)$ on an open bounded set $\mathcal{O} \subset \mathbb{R}^{2}$.

We will also need to consider the following functional spaces which are defined by periodic functions in the variable $y_{1} \in(0, L)$. Namely

$$
\begin{gathered}
L_{\#}^{p}\left(Y^{*}\right)=\left\{\varphi \in L^{p}\left(Y^{*}\right): \varphi\left(y_{1}, y_{2}\right) \text { is } L \text {-periodic in } y_{1}\right\}, \\
L_{\#}^{p}\left((0,1) \times Y^{*}\right)=\left\{\varphi \in L^{p}\left((0,1) \times Y^{*}\right): \varphi\left(x, y_{1}, y_{2}\right) \text { is } L \text {-periodic in } y_{1}\right\}, \\
W_{\#}^{1, p}\left(Y^{*}\right)=\left\{\varphi \in W^{1, p}\left(Y^{*}\right):\left.\varphi\right|_{\partial_{\text {left }} Y^{*}}=\left.\varphi\right|_{\partial_{\text {right }} Y^{*}}\right\} .
\end{gathered}
$$

If we denote by $[a]_{L}$ the unique integer number such that $a=[a]_{L} L+\{a\}_{L}$ where $\{a\}_{L} \in[0, L)$, then for each $\varepsilon>0$ and any $x \in \mathbb{R}$, we have

$$
x=\varepsilon^{\alpha}\left[\frac{x}{\varepsilon^{\alpha}}\right]_{L} L+\varepsilon^{\alpha}\left\{\frac{x}{\varepsilon^{\alpha}}\right\}_{L} \text { where }\left\{\frac{x}{\varepsilon^{\alpha}}\right\}_{L} \in[0, L) .
$$

Let us also denote

$$
I_{\varepsilon}=\operatorname{Int}\left(\bigcup_{k=0}^{N_{\varepsilon}}\left[k L \varepsilon^{\alpha},(k+1) L \varepsilon^{\alpha}\right]\right),
$$

where $N_{\varepsilon}$ is the largest integer such that $\varepsilon^{\alpha} L\left(N_{\varepsilon}+1\right) \leq 1$. We still set

$$
\begin{gathered}
\Lambda_{\varepsilon}=(0,1) \backslash I_{\varepsilon}=\left[\varepsilon^{\alpha} L\left(N_{\varepsilon}+1\right), 1\right), \\
R_{0}^{\varepsilon}=\left\{(x, y) \in \mathbb{R}^{2}: x \in I_{\varepsilon}, 0<y<\varepsilon^{\alpha} g\left(\frac{x}{\varepsilon^{\alpha}}\right)\right\}, \\
R_{1}^{\varepsilon}=\left\{(x, y) \in \mathbb{R}^{2}: x \in \Lambda_{\varepsilon}, 0<y<\varepsilon^{\alpha} g\left(\frac{x}{\varepsilon^{\alpha}}\right)\right\} .
\end{gathered}
$$

Observe that we have $\Lambda_{\varepsilon}=\emptyset$ if $\varepsilon^{\alpha} L\left(N_{\varepsilon}+1\right)=1$. In this case $R_{0}^{\varepsilon}=R^{\varepsilon}$ and $R_{1}^{\varepsilon}=\emptyset$.

The following well known inequalities will be needed throughout the paper (see [14]).

Proposition 2.1. Let $x, y \in \mathbb{R}^{n}$.

- If $p \geq 2$, then

$$
\left\langle|x|^{p-2} x-|y|^{p-2} y, x-y\right\rangle \geq c_{p}|x-y|^{p} .
$$

- If $1<p<2$, then

$$
\begin{aligned}
\left\langle|x|^{p-2} x-|y|^{p-2} y, x-y\right\rangle & \geq c_{p}|x-y|^{2}(|x|+|y|)^{p-2} \\
& \geq c_{p}|x-y|^{2}(1+|x|+|y|)^{p-2}
\end{aligned}
$$

Corollary 2.1.1. If for $1<p<\infty$, we denote by $a_{p}(s)=|s|^{p-2} s$ then $a_{p} \circ a_{p^{\prime}}(s)=s$ if $\frac{1}{p}+\frac{1}{p^{\prime}}=1$, that is, $a_{p}$ and $a_{p^{\prime}}$ are inverse functions. Hence,

- If $1<p^{\prime}<2$ (i.e, $p \geq 2$ ), then

$$
\left.|| u\right|^{p^{\prime}-2} u-|v|^{p^{\prime}-2} v|\leq c| u-\left.v\right|^{p^{\prime}-1} .
$$

- If $p^{\prime} \geq 2$ (i.e, $1<p<2$ ), then

$$
\begin{aligned}
\left.|| u\right|^{p^{\prime}-2} u-|v|^{p^{\prime}-2} v \mid & \leq c|u-v|(|u|+|v|)^{p^{\prime}-2} \\
& \leq c|u-v|(1+|u|+|v|)^{p^{\prime}-2} .
\end{aligned}
$$

Now, let us recall the definition to the unfolding operator and some of its properties. For proofs and details, see [5, 6]. 
Definition 2.2. Let $\varphi$ be a Lebesgue-measurable function in $R^{\varepsilon}$. The unfolding operator $T_{\varepsilon}$ acting on $\varphi$ is defined as the following function in $(0,1) \times Y^{*}$

$$
T_{\varepsilon} \varphi\left(x, y_{1}, y_{2}\right)=\left\{\begin{array}{l}
\varphi\left(\varepsilon^{\alpha}\left[\frac{x}{\varepsilon^{\alpha}}\right]_{L} L+\varepsilon^{\alpha} y_{1}, \varepsilon y_{2}\right), \text { for }\left(x, y_{1}, y_{2}\right) \in I_{\varepsilon} \times Y^{*} \\
0, \text { for }\left(x, y_{1}, y_{2}\right) \in \Lambda_{\varepsilon} \times Y^{*}
\end{array}\right.
$$

Proposition 2.3. The unfolding operator satifies the following properties:

1. $T_{\varepsilon}$ is linear;

2. $T_{\varepsilon}(\varphi \psi)=T_{\varepsilon}(\varphi) T_{\varepsilon}(\psi)$, for all $\varphi$, $\psi$ Lebesgue mesurable in $R^{\varepsilon}$;

3. $\forall \varphi \in L^{p}\left(R^{\varepsilon}\right), 1 \leq p \leq \infty$,

$$
T_{\varepsilon}(\varphi)\left(x,\left\{\frac{x}{\varepsilon^{\alpha}}\right\}_{L}, \frac{y}{\varepsilon}\right)=\varphi(x, y)
$$

for $(x, y) \in R_{0}^{\varepsilon}$.

4. Let $\varphi$ a Lebesgue mesurable function in $Y^{*}$ extended periodically in the first variable. Then, $\varphi^{\varepsilon}(x, y)=\varphi\left(\frac{x}{\varepsilon^{\alpha}}, \frac{y}{\varepsilon}\right)$ is mesurable in $R^{\varepsilon}$ and

$$
T_{\varepsilon}\left(\varphi^{\varepsilon}\right)\left(x, y_{1}, y_{2}\right)=\varphi\left(y_{1}, y_{2}\right), \forall\left(x, y_{1}, y_{2}\right) \in I_{\varepsilon} \times Y^{*} .
$$

Moreover, if $\varphi \in L^{p}\left(Y^{*}\right)$, then $\varphi^{\varepsilon} \in L^{p}\left(R^{\varepsilon}\right)$;

5. Let $\varphi^{\varepsilon} \in L^{1}\left(R^{\varepsilon}\right)$. Then,

$$
\begin{gathered}
\frac{1}{L} \int_{(0,1) \times Y^{*}} T_{\varepsilon}(\varphi)\left(x, y_{1}, y_{2}\right) d x d y_{1} d y_{2}=\frac{1}{\varepsilon} \int_{R_{0}^{\varepsilon}} \varphi(x, y) d x d y \\
=\frac{1}{\varepsilon} \int_{R^{\varepsilon}} \varphi(x, y) d x d y-\frac{1}{\varepsilon} \int_{R_{1}^{\varepsilon}} \varphi(x, y) d x d y
\end{gathered}
$$

6. $\forall \varphi \in L^{p}\left(R^{\varepsilon}\right), T_{\varepsilon}(\varphi) \in L^{p}\left((0,1) \times Y^{*}\right), 1 \leq p \leq \infty$. Moreover

$$
\left\|T_{\varepsilon}(\varphi)\right\|_{L^{p}\left((0,1) \times Y^{*}\right)}=\left(\frac{L}{\varepsilon}\right)^{\frac{1}{p}}\|\varphi\|_{L^{p}\left(R_{0}^{\varepsilon}\right)} \leq\left(\frac{L}{\varepsilon}\right)^{\frac{1}{p}}\|\varphi\|_{L^{p}\left(R^{\varepsilon}\right)} .
$$

If $p=\infty$,

$$
\left.\left\|\left.T_{\varepsilon}(\varphi)\right|_{L^{\infty}\left((0,1) \times Y^{*}\right)}=\right\| \varphi\right|_{L^{\infty}\left(R_{0}^{\varepsilon}\right)} \leq\|\varphi\|_{L^{\infty}\left(R^{\varepsilon}\right)} ;
$$

7. $\forall \varphi \in W^{1, p}\left(R^{\varepsilon}\right), 1 \leq p \leq \infty$,

$$
\partial_{y_{1}} T_{\varepsilon}(\varphi)=\varepsilon^{\alpha} T_{\varepsilon}\left(\partial_{x} \varphi\right) \text { and } \partial_{y_{2}} T_{\varepsilon}(\varphi)=\varepsilon T_{\varepsilon}\left(\partial_{y} \varphi\right) \text { a.e. in }(0,1) \times Y^{*} ;
$$

8. If $\varphi \in W^{1, p}\left(R^{\varepsilon}\right)$, then $T_{\varepsilon}(\varphi) \in L^{p}\left((0,1) ; W^{1, p}\left(Y^{*}\right)\right), 1 \leq p \leq \infty$. Besides, for $1 \leq p<\infty$, we have

$$
\begin{aligned}
\left\|\partial_{y_{1}} T_{\varepsilon}(\varphi)\right\|_{L^{p}\left((0,1) \times Y^{*}\right)} & =\varepsilon^{\alpha}\left(\frac{L}{\varepsilon}\right)^{\frac{1}{p}}\left\|\partial_{x} \varphi\right\|_{L^{p}\left(R_{0}^{\varepsilon}\right)} \leq \varepsilon^{\alpha}\left(\frac{L}{\varepsilon}\right)^{\frac{1}{p}}\left\|\partial_{x} \varphi\right\|_{L^{p}\left(R^{\varepsilon}\right)} \\
\left\|\partial_{y_{2}} T_{\varepsilon}(\varphi)\right\|_{L^{p}\left((0,1) \times Y^{*}\right)} & =\varepsilon\left(\frac{L}{\varepsilon}\right)^{\frac{1}{p}}\left\|\partial_{y} \varphi\right\|_{L^{p}\left(R_{0}^{\varepsilon}\right)} \leq \varepsilon\left(\frac{L}{\varepsilon}\right)^{\frac{1}{p}}\left\|\partial_{y} \varphi\right\|_{L^{p}\left(R^{\varepsilon}\right)} .
\end{aligned}
$$

If $p=\infty$,

$$
\begin{aligned}
& \left\|\partial_{y_{1}} T_{\varepsilon}(\varphi)\right\|_{L^{\infty}\left((0,1) \times Y^{*}\right)}=\varepsilon^{\alpha}\left\|\partial_{x} \varphi\right\|_{L^{\infty}\left(R_{0}^{\varepsilon}\right)} \leq \varepsilon^{\alpha}\left\|\partial_{x} \varphi\right\|_{L^{\infty}\left(R^{\varepsilon}\right)} \\
& \left\|\partial_{y_{2}} T_{\varepsilon}(\varphi)\right\|_{L^{\infty}\left((0,1) \times Y^{*}\right)}=\varepsilon\left\|\partial_{y} \varphi\right\|_{L^{\infty}\left(R_{0}^{\varepsilon}\right)} \leq \varepsilon\left\|\partial_{y} \varphi\right\|_{L^{\infty}\left(R^{\varepsilon}\right)} .
\end{aligned}
$$


Notice that, due to the order of the height of the thin domain the factor $1 / \varepsilon$ appears in properties 5 and 6 . Then, it makes sense to consider the following rescaled Lebesgue measure in the thin domains

$$
\rho_{\varepsilon}(\mathcal{O})=\frac{1}{\varepsilon}|\mathcal{O}|, \forall \mathcal{O} \subset R^{\varepsilon},
$$

which is widely considered in works involving thin domains. As a matter of fact, from now on, we use the following rescaled norms in the thin open sets

$$
\begin{aligned}
& \|\varphi \varphi\|_{L^{p}\left(R^{\varepsilon}\right)}=\varepsilon^{-1 / p}\|\varphi\|_{L^{p}\left(R^{\varepsilon}\right)}, \forall \varphi \in L^{p}\left(R^{\varepsilon}\right), 1 \leq p<\infty, \\
& \|\varphi\|_{W^{1, p}\left(R^{\varepsilon}\right)}=\varepsilon^{-1 / p}\|\varphi\|_{W^{1, p}\left(R^{\varepsilon}\right)} \forall \varphi \in W^{1, p}\left(R^{\varepsilon}\right), 1 \leq p<\infty .
\end{aligned}
$$

For completeness we may denote $\||\varphi|\|_{L^{\infty}\left(R^{\varepsilon}\right)}=\|\varphi\|_{L^{\infty}\left(R^{\varepsilon}\right)}$.

From property 6 , we have

$$
\left\|T_{\varepsilon}(\varphi)\right\|_{L^{p}\left((0,1) \times Y^{*}\right)} \leq L^{1 / p}\|\| \varphi \|_{L^{p}\left(R^{\varepsilon}\right)}, \quad 1 \leq p<\infty .
$$

Property 5 of Proposition 2.3 will be essential to pass to the limit when dealing with solutions of differential equations because it will allow us to transform any integral over the thin domain (which depends on the parameter $\varepsilon$ ) into an integral over the fixed set $(0,1) \times Y^{*}$. Notice that, in view of this property, we may say that the unfolding operator "almost preserves" the integral of the functions since the "integration defect" arises only from the unique cell which is not completely included in $R^{\varepsilon}$ and it is controlled by the integral on $R_{1}^{\varepsilon}$.

Therefore, an important concept for the unfolding method is the following property called unfolding criterion for integrals (u.c.i.).

Definition 2.4. A sequence $\left(\varphi_{\varepsilon}\right)$ satisfies the unfolding criterion for integrals (u.c.i) if

$$
\frac{1}{\varepsilon} \int_{R_{1}^{\varepsilon}}\left|\varphi_{\varepsilon}\right| d x d y \rightarrow 0 .
$$

Proposition 2.5. Let $\left(\varphi_{\varepsilon}\right)$ be a sequence in $L^{p}\left(R^{\varepsilon}\right), 1<p \leq \infty$ with the norm $\left\|\mid \varphi_{\varepsilon}\right\| \|_{L^{p}\left(R^{\varepsilon}\right)}$ uniformly bounded. Then, $\left(\varphi_{\varepsilon}\right)$ satisfies the (u.c.i).

Furthermore, let $\left(\psi_{\varepsilon}\right)$ be a sequence in $L^{q}\left(R^{\varepsilon}\right)$, also with $\left\|\left|\psi_{\varepsilon}\right|\right\|_{L^{q}\left(R^{\varepsilon}\right)}$ uniformly bounded, $\frac{1}{p}+\frac{1}{q}=\frac{1}{r}$, with $r>1$. Then, the product sequence $\left(\varphi_{\varepsilon} \psi_{\varepsilon}\right)$ satisfies (u.c.i).

If we still take $\phi \in L^{p^{\prime}}(0,1)$, then, the sequence $\varphi_{\varepsilon} \phi$ satifies (u.c.i).

Proposition 2.6. Let $\left(\varphi_{\varepsilon}\right)$ be a sequence in $L^{p}\left(R^{\varepsilon}\right), 1<p<\infty$ with $\left\|\left|\varphi_{\varepsilon}\right|\right\|_{L^{p}\left(R^{\varepsilon}\right)}$ uniformly bounded and let $\left(\psi_{\varepsilon}\right)$ be a sequence in $L^{p^{\prime}}\left(R^{\varepsilon}\right)$ set as follows

$$
\psi_{\varepsilon}(x, y)=\psi\left(\frac{x}{\varepsilon^{\alpha}}, \frac{y}{\varepsilon}\right),
$$

where $\psi \in L^{p^{\prime}}\left(Y^{*}\right)$. Then, $\left(\varphi_{\varepsilon} \psi_{\varepsilon}\right)$ satisfies (u.c.i).

Now, let us recall some convergence properties of the unfolding operator as $\varepsilon$ goes to zero.

Theorem 2.7. For a measurable function $f$ on $Y^{*}$, L-periodic in its first variable and extended by periodicity to $\left\{(x, y) \in \mathbb{R}^{2}: x \in \mathbb{R}, 0<y<g(x)\right\}$, define the sequence $\left(f_{\varepsilon}\right)$ by

$$
f^{\varepsilon}(x, y)=f\left(\frac{x}{\varepsilon^{\alpha}}, \frac{y}{\varepsilon}\right)
$$

a.e. for

$$
(x, y) \in\left\{(x, y) \in \mathbb{R}^{2}: x \in \mathbb{R}, 0<y<\varepsilon g\left(\frac{x}{\varepsilon^{\alpha}}\right)\right\} .
$$

Then

$$
\left.T_{\varepsilon} f^{\varepsilon}\right|_{(0,1)}\left(x, y_{1}, y_{2}\right)=\left\{\begin{array}{l}
f\left(y_{1}, y_{2}\right), \text { for }\left(x, y_{1}, y_{2}\right) \in I_{\varepsilon} \times Y^{*}, \\
0, \text { for }\left(x, y_{1}, y_{2}\right) \in \Lambda_{\varepsilon} \times Y^{*} .
\end{array}\right.
$$

Moreover, if $f \in L_{\#}^{p}\left(Y^{*}\right)$, then

$$
T_{\varepsilon} f^{\varepsilon} \rightarrow f
$$

strongly in $L_{\#}^{p}\left((0,1) \times Y^{*}\right)$. 
Proposition 2.8. Let $f \in L^{p}\left((0,1) ; L_{\#}^{p}\left(Y^{*}\right)\right)$ and extend it periodically in $y_{1}$-direction defining

$$
f^{\varepsilon}(x, y):=f\left(x, \frac{x}{\varepsilon^{\alpha}}, \frac{y}{\varepsilon}\right) \in L^{p}\left(R^{\varepsilon}\right) .
$$

Then,

$$
T_{\varepsilon} f^{\varepsilon} \rightarrow f \text { strongly in } L^{p}\left((0,1) \times Y^{*}\right) \text {. }
$$

Proof. It follows from Theorem 2.7 and the density of the tensor product $C_{0}^{\infty}(0,1) \otimes L_{\#}^{p}\left(Y^{*}\right)$ in $L^{p}\left((0,1) ; L_{\#}^{p}\left(Y^{*}\right)\right)$.

Remark 2.1. Using Proposition 2.8, we also have that, if $T_{\varepsilon} f^{\varepsilon} \rightarrow f$ strongly in $L^{p}\left((0,1) \times Y^{*}\right)$, then

$$
T_{\varepsilon}\left(\left|f^{\varepsilon}\right|^{p-2} f^{\varepsilon}\right) \rightarrow|f|^{p-2} f \text { strongly in } L^{p^{\prime}}\left((0,1) \times Y^{*}\right) .
$$

In particular, we can take $f^{\varepsilon}$ as in (8).

Proposition 2.9. Let $\varphi \in L^{p}(0,1), 1 \leq p<\infty$. Then, considering $\varphi$ as a function defined in $R^{\varepsilon}$, we have

$$
T_{\varepsilon} \varphi \rightarrow \varphi \text { strongly in } L^{p}\left((0,1) \times Y^{*}\right) .
$$

Proposition 2.10. Let $\left(\varphi_{\varepsilon}\right)$ be a sequence in $L^{p}(0,1), 1 \leq p<\infty$, such that

$$
\varphi_{\varepsilon} \rightarrow \varphi \text { strongly in } L^{p}(0,1) \text {. }
$$

Then,

$$
T_{\varepsilon} \varphi_{\varepsilon} \rightarrow \varphi \text { strongly in } L^{p}\left((0,1) \times Y^{*}\right) \text {. }
$$

Next, we recall a convergence result which does not depend on the value of the parameter $\alpha$. To do that, we first introduce a suitable decomposition to functions $\varphi \in W^{1, p}\left(R^{\varepsilon}\right)$ where the geometry of the thin domains plays a crucial role. We write $\varphi(x, y)=V(x)+\varphi_{r}(x, y)$ where $V$ is defined as follows

$$
V(x):=\frac{1}{\varepsilon g_{0}} \int_{0}^{\varepsilon g_{0}} \varphi(x, s) d s \quad \text { a.e. } x \in(0,1) .
$$

We set $\varphi_{r}(x, y) \equiv \varphi(x, y)-V(x)$.

Proposition 2.11. Let $\left(\varphi_{\varepsilon}\right) \subset W^{1, p}\left(R^{\varepsilon}\right), 1<p<\infty$, with $\left\|\left|\varphi_{\varepsilon}\right|\right\|_{W^{1, p}\left(R^{\varepsilon}\right)}$ uniformly bounded and $V_{\varepsilon}(x)$ defined as in 10$]$. Then, there exists a function $\varphi \in W^{1, p}(0,1)$ such that, up to subsequences

$$
\begin{aligned}
& V_{\varepsilon} \rightarrow \varphi \text { weakly in } W^{1, p}(0,1) \text { and strongly in } L^{p}(0,1), \\
& T_{\varepsilon} V_{\varepsilon} \rightarrow \varphi \text { strongly in } L^{p}\left((0,1) \times Y^{*}\right), \\
& \left\|\varphi_{\varepsilon}-V_{\varepsilon}\right\|_{L^{p}\left(R^{\varepsilon}\right)} \rightarrow 0 \\
& \left\|\varphi_{\varepsilon}-\varphi\right\| \|_{L^{p}\left(R^{\varepsilon}\right)} \rightarrow 0 \\
& T_{\varepsilon} \varphi_{\varepsilon} \rightarrow \varphi \text { weakly in } L^{p}\left((0,1) ; W^{1, p}\left(Y^{*}\right)\right) \\
& T_{\varepsilon} \varphi_{\varepsilon} \rightarrow \varphi \text { strongly in } L^{p}\left((0,1) \times Y^{*}\right) .
\end{aligned}
$$

Furthermore, there exists $\bar{\varphi} \in L^{p}\left((0,1) \times Y^{*}\right)$ with $\partial_{y_{2}} \bar{\varphi} \in L^{p}\left((0,1) \times Y^{*}\right)$ such that, up to subsequences

$$
\begin{aligned}
& \frac{1}{\varepsilon} T_{\varepsilon}\left(\varphi_{r}^{\varepsilon}\right) \rightarrow \bar{\varphi} \text { weakly in } L^{p}\left((0,1) \times Y^{*}\right) \text {, and } \\
& T_{\varepsilon}\left(\partial_{y} \varphi_{\varepsilon}\right) \rightarrow \partial_{y_{2}} \bar{\varphi} \text { weakly in } L^{p}\left((0,1) \times Y^{*}\right)
\end{aligned}
$$

where $\varphi_{r}^{\varepsilon} \equiv \varphi_{\varepsilon}-V_{\varepsilon}$.

Now, let us recall a compactness result which allows us to identify the limit of the image of the gradient of a uniformly bounded sequence by the unfolding operator method as $0<\alpha \leq 1$ in (1). 
Theorem 2.12. Let $\left(\varphi_{\varepsilon}\right) \subset W^{1, p}\left(R^{\varepsilon}\right), 1<p<\infty$, with $\left\|\left|\varphi_{\varepsilon}\right|\right\|_{W^{1, p}\left(R^{\varepsilon}\right)}$ uniformly bounded.

Then, there exists $\varphi \in W^{1, p}(0,1)$ and $\varphi_{1} \in L^{p}\left((0,1) ; W_{\#}^{1, p}\left(Y^{*}\right)\right)$ such that (up to a subsequence)

a) if $\alpha=1$, we have

$$
\begin{aligned}
& T_{\varepsilon} \varphi_{\varepsilon} \rightarrow \varphi \text { weakly in } L^{p}\left((0,1) ; W^{1, p}\left(Y^{*}\right)\right), \\
& T_{\varepsilon} \partial_{x} \varphi_{\varepsilon} \rightarrow \partial_{x} \varphi+\partial_{y_{1}} \varphi_{1} \text { weakly in } L^{p}\left((0,1) \times Y^{*}\right), \\
& T_{\varepsilon} \partial_{y} \varphi_{\varepsilon} \rightarrow \partial_{y_{2}} \varphi_{1} \text { weakly in } L^{p}\left((0,1) \times Y^{*}\right) .
\end{aligned}
$$

b) If $\alpha<1$, we obtain $\partial_{y_{2}} \varphi_{1}=0$ and

$$
\begin{aligned}
& T_{\varepsilon} \varphi_{\varepsilon} \rightarrow \varphi, \text { weakly in } L^{p}\left((0,1) ; W^{1, p}\left(Y^{*}\right)\right), \\
& T_{\varepsilon} \partial_{x} \varphi_{\varepsilon} \rightarrow \partial_{x} \varphi+\partial_{y_{1}} \varphi_{1} \text {, weakly in } L^{p}\left((0,1) \times Y^{*}\right) .
\end{aligned}
$$

Proof. See [6, Theorem 3.1 and 4.1] respectively.

Finally, we obtain uniform boundedness to the solutions of the $p$-Laplacian problem (2) for any value of $\alpha>0$.

Proposition 2.13. Consider the variational formulation of our problem:

$$
\int_{R^{\varepsilon}}\left\{\left|\nabla u_{\varepsilon}\right|^{p-2} \nabla u_{\varepsilon} \nabla \varphi+\left|u_{\varepsilon}\right|^{p-2} u_{\varepsilon} \varphi\right\} d x d y=\int_{R^{\varepsilon}} f^{\varepsilon} \varphi d x d y, \varphi \in W^{1, p}\left(R^{\varepsilon}\right),
$$

where $f^{\varepsilon}$ satisfies

$$
\|\left.\left|f^{\varepsilon}\right|\right|_{L^{p^{\prime}}\left(R^{\varepsilon}\right)} \leq c
$$

for some positive constant $c$ independent of $\varepsilon>0$. Then,

$$
\begin{gathered}
\left\|\left|u_{\varepsilon}\right|\right\|_{L^{p}\left(R^{\varepsilon}\right)} \leq c, \quad\left\|u_{\varepsilon} \mid\right\|_{W^{1, p}\left(R^{\varepsilon}\right)} \leq c, \\
\|\|\left|\nabla u_{\varepsilon}\right|^{p-2} \nabla u_{\varepsilon} \mid \|_{L^{p^{\prime}\left(R^{\varepsilon}\right)}} \leq c .
\end{gathered}
$$

Proof. Take $\varphi=u_{\varepsilon}$ in 11. Then,

$$
\left\|u_{\varepsilon}\right\|_{W^{1, p}\left(R^{\varepsilon}\right)}^{p}=\int_{R^{\varepsilon}}\left\{\left|\nabla u_{\varepsilon}\right|^{p}+\left|u_{\varepsilon}\right|^{p}\right\} d x d y \leq\left\|f^{\varepsilon}\right\|_{L^{p^{\prime}\left(R^{\varepsilon}\right)}}\left\|u_{\varepsilon}\right\|_{L^{p}\left(R^{\varepsilon}\right)} .
$$

Hence,

$$
\left\|u_{\varepsilon}\right\|_{W^{1, p}\left(R^{\varepsilon}\right)} \leq c .
$$

Therefore, the sequence $u_{\varepsilon}$ and $\left|\nabla u_{\varepsilon}\right|^{p-2} \nabla u_{\varepsilon}$, are respectively bounded in $L^{p}\left(R^{\varepsilon}\right)$ and $\left(L^{p^{\prime}}\left(R^{\varepsilon}\right)\right)^{2}$ under the norm $|\|\cdot \mid\|$.

\section{The resonant case: $\alpha=1$.}

In this section, we use the results on the Unfolding Operator described in Section 2 in order to pass to the limit in problem (2) assuming $\alpha=1$. Notice that this case is called resonant since the amplitude and period of the oscillation are of the same order as the thickness of the thin domain.

Thus, we consider here in this section, the following two-dimensional thin domain family

$$
R^{\varepsilon}=\left\{(x, y) \in \mathbb{R}^{2}: 0<x<1,0<y<\varepsilon g\left(\frac{x}{\varepsilon}\right)\right\} .
$$

with $g$ satisfying hypothesis $\left(\mathbf{H}_{\mathbf{g}}\right)$.

We have the following result. 
Theorem 3.1. Let $u_{\varepsilon}$ be the solution of problem (2) with $f^{\varepsilon}$ satisfying

$$
\||| f^{\varepsilon}||_{L^{p^{\prime}\left(R^{\varepsilon}\right)}} \leq c
$$

for $c>0$ independent of $\varepsilon>0$. Suppose also that

$$
T_{\varepsilon} f^{\varepsilon} \rightarrow \hat{f} \text { weakly in } L^{p^{\prime}}\left((0,1) \times Y^{*}\right) .
$$

Then, there exists $\left(u, u_{1}\right) \in W^{1, p}(0,1) \times L^{p}\left((0,1) ; W_{\#}^{1, p}\left(Y^{*}\right)\right)$ such that

$$
\left\{\begin{array}{l}
T_{\varepsilon} u_{\varepsilon} \rightarrow u \text { weakly in } L^{p}\left((0,1) ; W^{1, p}\left(Y^{*}\right)\right), \\
T_{\varepsilon}\left(\partial_{x} u_{\varepsilon}\right) \rightarrow \partial_{x} u+\partial_{y_{1}} u_{1}\left(x, y_{1}, y_{2}\right) \text { weakly in } L^{p}\left((0,1) ; W^{1, p}\left(Y^{*}\right)\right), \\
T_{\varepsilon}\left(\partial_{y} u_{\varepsilon}\right) \rightarrow \partial_{y_{2}} u_{1}\left(x, y_{1}, y_{2}\right) \text { weakly in } L^{p}\left((0,1) ; W^{1, p}\left(Y^{*}\right)\right),
\end{array}\right.
$$

and $u$ is the solution of the problem

$$
\int_{0}^{1}\left(q\left|\partial_{x} u\right|^{p-2} \partial_{x} u \partial_{x} \varphi+\left|\partial_{x} u\right|^{p-2} \partial_{x} u \varphi\right) d x=\int_{0}^{1} \bar{f} \varphi d x
$$

where

$$
\begin{gathered}
\bar{f}=\frac{1}{\left|Y^{*}\right|} \int_{Y^{*}} \hat{f} d y_{1} d y_{2}, \\
q=\frac{1}{\left|Y^{*}\right|} \int_{Y^{*}}|\nabla v|^{p-2} \partial_{y_{1}} v d y_{1} d y_{2},
\end{gathered}
$$

and $v$ is the solution of the auxiliar problem

$$
\begin{gathered}
\int_{Y^{*}}|\nabla v|^{p-2} \nabla v \nabla \varphi d y_{1} d y_{2}=0, \quad \forall \varphi \in W_{\#, 0}^{1, p}\left(Y^{*}\right), \\
\left(v-y_{1}\right) \in W_{\#, 0}^{1, p}\left(Y^{*}\right)
\end{gathered}
$$

where $W_{\#, 0}^{1, p}\left(Y^{*}\right)$ denotes the subspace of $W_{\#}^{1, p}\left(Y^{*}\right)$ of functions with zero average.

Moreover,

$$
u^{\prime}(x) \nabla_{y} v\left(y_{1}, y_{2}\right)=\left(u^{\prime}(x), 0\right)+\nabla_{y} u_{1}\left(x, y_{1}, y_{2}\right)
$$

where $\nabla_{y} \cdot=\left(\partial_{y_{1}} \cdot \partial_{y_{2}} \cdot\right)$.

Proof. From Proposition 2.3, we can rewrite (11) as

$$
\begin{gathered}
\int_{(0,1) \times Y^{*}} T_{\varepsilon}\left(\left|\nabla u_{\varepsilon}\right|^{p-2} \nabla u_{\varepsilon}\right) T_{\varepsilon} \nabla \varphi d x d y_{1} d y_{2}+\frac{L}{\varepsilon} \int_{R_{1}^{\varepsilon}}\left|\nabla u_{\varepsilon}\right|^{p-2} \nabla u_{\varepsilon} \nabla \varphi d x d y \\
+\int_{(0,1) \times Y^{*}} T_{\varepsilon}\left(\left|u_{\varepsilon}\right|^{p-2} u_{\varepsilon}\right) T_{\varepsilon} \varphi d x d y_{1} d y_{2}+\frac{L}{\varepsilon} \int_{R_{1}^{\varepsilon}}\left|u_{\varepsilon}\right|^{p-2} u_{\varepsilon} \varphi d x d y \\
=\int_{(0,1) \times Y^{*}} T_{\varepsilon} f^{\varepsilon} T_{\varepsilon} \varphi d x d y_{1} d y_{2}+\frac{L}{\varepsilon} \int_{R_{1}^{\varepsilon}} f^{\varepsilon} \varphi d x d y .
\end{gathered}
$$

By Proposition 2.13 and Theorem 2.12, there exist

$$
u \in W^{1, p}(0,1), \quad u_{1} \in L^{p}\left((0,1) ; W_{\#}^{1, p}\left(Y^{*}\right)\right) \quad \text { and } \quad a_{0} \in L^{p}\left((0,1) \times Y^{*}\right)^{2}
$$

such that, up to subsequences,

$$
\left\{\begin{array}{l}
T_{\varepsilon} u_{\varepsilon} \rightarrow u \text { strongly in } L^{p}\left((0,1) \times Y^{*}\right) \\
T_{\varepsilon}\left(\partial_{x} u_{\varepsilon}\right) \rightarrow \partial_{x} u+\partial_{y_{1}} u_{1}\left(x, y_{1}, y_{2}\right) \text { weakly in } L^{p}\left((0,1) ; W^{1, p}\left(Y^{*}\right)\right) \\
T_{\varepsilon}\left(\partial_{y} u_{\varepsilon}\right) \rightarrow \partial_{y_{2}} u_{1}\left(x, y_{1}, y_{2}\right) \text { weakly in } L^{p}\left((0,1) ; W^{1, p}\left(Y^{*}\right)\right) \\
T_{\varepsilon}\left(\left|\nabla u_{\varepsilon}\right|^{p-2} \nabla u_{\varepsilon}\right) \rightarrow a_{0} \text { weakly in } L^{p}\left((0,1) \times Y^{*}\right)^{2}
\end{array}\right.
$$


We still have from Remark 2.1 that

$$
\left|T_{\varepsilon} u_{\varepsilon}\right|^{p-2} T_{\varepsilon} u_{\varepsilon} \rightarrow|u|^{p-2} u \text { strongly in } L^{p^{\prime}}\left((0,1) \times Y^{*}\right) .
$$

Hence, passing to the limit in 17 for test functions $\varphi \in W^{1, p}(0,1)$, we get

$$
\int_{(0,1) \times Y^{*}} a_{0} \nabla \varphi+|u|^{p-2} u \varphi d x d y_{1} d y_{2}=\int_{(0,1) \times Y^{*}} \hat{f} \varphi d x d y_{1} d y_{2} .
$$

Let $\phi \in C_{0}^{\infty}(0,1)$ and $\psi \in W_{\#}^{1, p}\left(Y^{*}\right)$. Define the sequence

$$
v_{\varepsilon}(x, y)=\varepsilon \phi(x) \psi\left(\frac{x}{\varepsilon}, \frac{y}{\varepsilon}\right) .
$$

We apply the unfolding operator in this sequence and obtain

$$
\begin{array}{r}
T_{\varepsilon} v_{\varepsilon} \rightarrow 0 \text { strongly in } L^{p}\left((0,1) \times Y^{*}\right), \\
T_{\varepsilon}\left(\partial_{x} v_{\varepsilon}\right) \rightarrow \phi \partial_{y_{1}} \psi \text { strongly in } L^{p}\left((0,1) \times Y^{*}\right), \\
T_{\varepsilon}\left(\partial_{y} v_{\varepsilon}\right) \rightarrow \phi \partial_{y_{2}} \psi \text { strongly in } L^{p}\left((0,1) \times Y^{*}\right) .
\end{array}
$$

Thus, taking $v_{\varepsilon}$ as a test function in (17), we obtain at $\varepsilon=0$ that

$$
\int_{(0,1) \times Y^{*}} a_{0} \phi(x) \nabla_{y} \psi d x d y_{1} d y_{2}=0 .
$$
that

Hence, we get from 20 and the density of the tensor product $C_{0}^{\infty}(0,1) \otimes W_{\#}^{1, p}\left(Y^{*}\right)$ in $L^{p}\left((0,1) ; W_{\#}^{1, p}\left(Y^{*}\right)\right)$

$$
\int_{(0,1) \times Y^{*}} a_{0} \nabla_{y} \psi d x d y_{1} d y_{2}=0, \quad \forall \psi \in L^{p}\left((0,1) ; W_{\#}^{1, p}\left(Y^{*}\right)\right) .
$$

Now, let us identify $a_{0}$. For this sake, let $u_{1} \in L^{p}\left((0,1) ; W_{\#}^{1, p}\left(Y^{*}\right)\right)$ and $u \in W^{1, p}(0,1)$ be the ones obtained in (18). Extend $\nabla_{y} u_{1}$ in the $y_{1}$-direction, and then define the sequence

$$
W_{\varepsilon}(x, y)=\left(\partial_{x} u(x), 0\right)+\nabla_{y} u_{1}\left(x, \frac{x}{\varepsilon}, \frac{y}{\varepsilon}\right) .
$$

See that $W_{\varepsilon} \in L^{p}\left(R^{\varepsilon}\right) \times L^{p}\left(R^{\varepsilon}\right)$. Also, due to Proposition 2.8, we have

$$
\begin{gathered}
T_{\varepsilon} W_{\varepsilon} \rightarrow\left(\partial_{x} u, 0\right)+\nabla_{y} u_{1}, \quad \text { and } \\
T_{\varepsilon}\left(\left|W_{\varepsilon}\right|^{p-2} W_{\varepsilon}\right) \rightarrow\left|\left(\partial_{x} u, 0\right)+\nabla_{y} u_{1}\right|^{p-2}\left[\left(\partial_{x} u, 0\right)+\nabla_{y} u_{1}\right]
\end{gathered}
$$

strongly in $L^{p}\left((0,1) \times Y^{*}\right)^{2}$ and $L^{p^{\prime}}\left((0,1) \times Y^{*}\right)^{2}$, respectively.

We need to prove that

$$
\int_{(0,1) \times Y^{*}}\left[\left|\nabla u+\nabla_{y} u_{1}\right|^{p-2}\left(\nabla u+\nabla_{y} u_{1}\right)-a_{0}\right] \varphi d x d y_{1} d y_{2}=0
$$

for all $\varphi \in C_{0}^{\infty}\left((0,1) \times Y^{*}\right) \times C_{0}^{\infty}\left((0,1) \times Y^{*}\right)$ in order to identify $a_{0}$.

For this sake, let us first prove that the right hand side of

$$
0 \leq \int_{(0,1) \times Y^{*}} T_{\varepsilon}\left[\left|\nabla u_{\varepsilon}\right|^{p-2} \nabla u_{\varepsilon}-\left|W_{\varepsilon}\right|^{p-2} W_{\varepsilon}\right] T_{\varepsilon}\left(\nabla u_{\varepsilon}-W_{\varepsilon}\right) d x d y_{1} d y_{2}
$$

converges to zero. Notice that by the monotonicity of $|\cdot|^{p-2} \cdot$ (see Proposition 2.1) the inequality above is obtained. To pass to the limit in (24), we first use a distributive in the integral. 
Using (17), denoting $d Y=d y_{1} d y_{2}$ and using (18), we get that

$$
\begin{gathered}
\lim _{\varepsilon \rightarrow 0} \int_{(0,1) \times Y^{*}} T_{\varepsilon}\left(\left|\nabla u_{\varepsilon}\right|^{p-2} \nabla u_{\varepsilon}\right) T_{\varepsilon}\left(\nabla u_{\varepsilon}\right) d x d Y \\
=\lim _{\varepsilon \rightarrow 0}\left[\int_{(0,1) \times Y^{*}} T_{\varepsilon} f^{\varepsilon} T_{\varepsilon} u_{\varepsilon} d x d Y+\frac{L}{\varepsilon} \int_{R_{1}^{\varepsilon}} f^{\varepsilon} u_{\varepsilon} d x d y\right. \\
\left.-\int_{(0,1) \times Y^{*}} T_{\varepsilon}\left(\left|u_{\varepsilon}\right|^{p-2} u_{\varepsilon}\right) T_{\varepsilon} u_{\varepsilon} d x d Y-\frac{L}{\varepsilon} \int_{R_{1}^{\varepsilon}}\left|u_{\varepsilon}\right|^{p-2} u_{\varepsilon} u_{\varepsilon} d x d y\right]= \\
\int_{(0,1) \times Y^{*}}\left(\hat{f}-|u|^{p-2} u\right) u d x d Y .
\end{gathered}
$$

Consequently, we get from 190 that

$$
\lim _{\varepsilon \rightarrow 0} \int_{(0,1) \times Y^{*}} T_{\varepsilon}\left(\left|\nabla u_{\varepsilon}\right|^{p-2} \nabla u_{\varepsilon}\right) T_{\varepsilon}\left(\nabla u_{\varepsilon}\right) d x d Y=\int_{(0,1) \times Y^{*}} a_{0} \nabla u d x d Y .
$$

On the other hand, due to (18), 23) and $(21)$, we get

$$
\begin{gathered}
\lim _{\varepsilon \rightarrow 0} \int_{(0,1) \times Y^{*}} T_{\varepsilon}\left(\left|\nabla u_{\varepsilon}\right|^{p-2} \nabla u_{\varepsilon}\right) T_{\varepsilon}\left(W_{\varepsilon}\right) d x d Y \\
=\int_{(0,1) \times Y^{*}} a_{0}\left(\nabla u+\nabla_{y} u_{1}\right) d x d Y \\
=\int_{(0,1) \times Y^{*}} a_{0} \nabla u d x d Y .
\end{gathered}
$$

Finally, we have

$$
\lim _{\varepsilon \rightarrow 0} \int_{(0,1) \times Y^{*}} T_{\varepsilon}\left(\left|W_{\varepsilon}\right|^{p-2} W_{\varepsilon}\right) T_{\varepsilon}\left(\nabla u_{\varepsilon}-W_{\varepsilon}\right) d x d Y=0,
$$

by (23) and (18). Indeed, we have $T_{\varepsilon}\left(\nabla u_{\varepsilon}-W_{\varepsilon}\right) \rightarrow 0$ weakly in $L^{p}\left((0,1) \times Y^{*}\right)$.

Thus, from (25), 26) and (27), we can pass to the limit in (24) to get

$$
\int_{(0,1) \times Y^{*}} T_{\varepsilon}\left[\left|\nabla u_{\varepsilon}\right|^{p-2} \nabla u_{\varepsilon}-\left|W_{\varepsilon}\right|^{p-2} W_{\varepsilon}\right] T_{\varepsilon}\left(\nabla u_{\varepsilon}-W_{\varepsilon}\right) d x d Y \rightarrow 0 .
$$

Suppose $p \geq 2$. Then, by Proposition 2.1, we have

$$
\begin{aligned}
& \int_{(0,1) \times Y^{*}}\left|T_{\varepsilon} \nabla u_{\varepsilon}-T_{\varepsilon} W_{\varepsilon}\right|^{p} d x d Y \\
\leq & c \int_{(0,1) \times Y^{*}} T_{\varepsilon}\left(\left|\nabla u_{\varepsilon}\right|^{p-2} \nabla u_{\varepsilon}-\left|W_{\varepsilon}\right|^{p-2} W_{\varepsilon}\right)\left(T_{\varepsilon} \nabla u_{\varepsilon}-T_{\varepsilon} W_{\varepsilon}\right) d x d Y \\
\rightarrow & 0 \quad \text { as } \varepsilon \rightarrow 0 .
\end{aligned}
$$

If $1<p \leq 2$, we have, using a Hölder's inequality for the exponent $\frac{2}{p}$ (and its conjugate $\frac{2}{2-p}$ ) and 
Proposition 2.1.

$$
\begin{aligned}
& \int_{(0,1) \times Y^{*}}\left|T_{\varepsilon} \nabla u_{\varepsilon}-T_{\varepsilon} W_{\varepsilon}\right|^{p} d x d Y \\
= & \int_{(0,1) \times Y^{*}}\left|T_{\varepsilon} \nabla u_{\varepsilon}-T_{\varepsilon} W_{\varepsilon}\right|^{p} \frac{\left(1+\left|T_{\varepsilon} \nabla u_{\varepsilon}\right|+\left|T_{\varepsilon} W_{\varepsilon}\right|\right)^{\frac{(p-2) p}{2}}}{\left(1+\left|T_{\varepsilon} \nabla u_{\varepsilon}\right|+\left|T_{\varepsilon} W_{\varepsilon}\right|\right)^{\frac{(p-2) p}{2}}} d x d Y \\
\leq & \left(\int_{(0,1) \times Y^{*}}\left|T_{\varepsilon} \nabla u_{\varepsilon}-T_{\varepsilon} W_{\varepsilon}\right|^{2}\left(1+\left|T_{\varepsilon} \nabla u_{\varepsilon}\right|+\left|T_{\varepsilon} W_{\varepsilon}\right|\right)^{(p-2)} d x d Y\right)^{p / 2} \\
& \left(\int_{(0,1) \times Y^{*}}\left(1+\left|T_{\varepsilon} \nabla u_{\varepsilon}\right|+\left|T_{\varepsilon} W_{\varepsilon}\right|\right)^{p} d x d Y\right)^{(2-p) / 2} \\
\leq & c\left[\int_{(0,1) \times Y^{*}} T_{\varepsilon}\left(\left|\nabla u_{\varepsilon}\right|^{p-2} \nabla u_{\varepsilon}-\left|W_{\varepsilon}\right|^{p-2} W_{\varepsilon}\right)\left(T_{\varepsilon} \nabla u_{\varepsilon}-T_{\varepsilon} W_{\varepsilon}\right) d x d Y\right]^{p / 2} \\
\rightarrow & 0 .
\end{aligned}
$$

Now, let us prove

$$
\int_{(0,1) \times Y^{*}} T_{\varepsilon}\left(\left|\nabla u_{\varepsilon}\right|^{p-2} \nabla u_{\varepsilon}-\left|W_{\varepsilon}\right|^{p-2} W_{\varepsilon}\right) \varphi d x d Y \rightarrow 0
$$

for any test function $\varphi \in C_{0}^{\infty}\left((0,1) \times Y^{*}\right) \times C_{0}^{\infty}\left((0,1) \times Y^{*}\right)$.

First, consider $p \geq 2$. Therefore, from Corollary (2.1.1) and Young inequality, we get

$$
\begin{aligned}
& \int_{(0,1) \times Y^{*}} T_{\varepsilon}\left(\left|\nabla u_{\varepsilon}\right|^{p-2} \nabla u_{\varepsilon}-\left|W_{\varepsilon}\right|^{p-2} W_{\varepsilon}\right) \varphi d x d Y \\
\leq & \left.c \int_{(0,1) \times Y^{*}}|| T_{\varepsilon} \nabla u_{\varepsilon}\right|^{p-2} T_{\varepsilon} \nabla u_{\varepsilon}-\left|T_{\varepsilon} W_{\varepsilon}\right|^{p-2} T_{\varepsilon} W_{\varepsilon} \mid d x d Y \\
\leq & c \int_{(0,1) \times Y^{*}}\left(1+\left|T_{\varepsilon} \nabla u_{\varepsilon}\right|+\left|T_{\varepsilon} W_{\varepsilon}\right|\right)^{p-2}\left|T_{\varepsilon}\left(\nabla u_{\varepsilon}-W_{\varepsilon}\right)\right| d x d Y \\
\leq & c\left(\int_{(0,1) \times Y^{*}}\left(1+\left|T_{\varepsilon} \nabla u_{\varepsilon}\right|+\left|T_{\varepsilon} W_{\varepsilon}\right|\right)^{p} d x d Y\right)^{1 / p^{\prime}} \\
& \quad\left(\int_{(0,1) \times Y^{*}}\left|T_{\varepsilon} \nabla u_{\varepsilon}-T_{\varepsilon} W_{\varepsilon}\right|^{p} d x d Y\right)^{1 / p} \\
\rightarrow & 0,
\end{aligned}
$$

by convergence 29 .

For $1<p<2$, we perform analogous arguments. Using Corollary 2.1.1, a Hölder's inequality and the convergence $(30)$, one gets

$$
\begin{aligned}
& \int_{(0,1) \times Y^{*}} T_{\varepsilon}\left(\left|\nabla u_{\varepsilon}\right|^{p-2} \nabla u_{\varepsilon}-\left|W_{\varepsilon}\right|^{p-2} W_{\varepsilon}\right) \varphi d x d Y \\
\leq & c \int_{(0,1) \times Y^{*}}\left|T_{\varepsilon} \nabla u_{\varepsilon}-T_{\varepsilon} W_{\varepsilon}\right|^{p-1} d x d Y \\
= & c\left(\int_{(0,1) \times Y^{*}}\left|T_{\varepsilon} \nabla u_{\varepsilon}-T_{\varepsilon} W_{\varepsilon}\right|^{p} d x d Y\right)^{1 / p^{\prime}} \\
\rightarrow & 0 .
\end{aligned}
$$

Thus, for any $p>1$ and $\varphi \in C_{0}^{\infty}\left((0,1) \times Y^{*}\right) \times C_{0}^{\infty}\left((0,1) \times Y^{*}\right)$, we have

$$
\int_{(0,1) \times Y^{*}}\left(a_{0}-\left|\nabla u+\nabla_{y} u_{1}\right|^{p-2}\left(\nabla u+\nabla_{y} u_{1}\right)\right) \varphi d x d Y=0 .
$$


Finally, let us associate $a_{0}$ with the auxiliary problem (15). We first rewrite (21) as

$$
\int_{(0,1) \times Y^{*}}\left|\nabla u+\nabla_{y} u_{1}\right|^{p-2}\left(\nabla u+\nabla_{y} u_{1}\right) \nabla_{y} \psi d x d Y=0
$$

for any $\psi \in L^{p}\left((0,1) ; W_{\#}^{1, p}\left(Y^{*}\right)\right)$.

From Minty-Browder Theorem, we have that (32) sets a well posed problem, and then, for each $u \in W^{1, p}(0,1)$, possesses an unique solution $u_{1} \in L^{p}\left((0,1) ; W_{\#}^{1, p}\left(Y^{*}\right) / \mathbb{R}\right)$. Notice that $W_{\#}^{1, p}\left(Y^{*}\right) / \mathbb{R}$ is identified with the closed subspace of $W_{\#}^{1, p}\left(Y^{*}\right)$ consisting of all its functions with zero average.

We might mention that multiplying the solution $v$ of the equation (15) by $u^{\prime}$, then $u^{\prime} v$ depends on $x$ and belongs to the space $L^{p}\left((0,1) ; W_{\#}^{1, p}\left(Y^{*}\right) / \mathbb{R}\right)$. Multiplying 15$)$ by a function $\left|\partial_{x} u\right|^{p-2} \partial_{x} u$ and by $\phi \in C_{0}^{\infty}(0,1)$, and integrating in $(0,1)$, we get

$$
\int_{(0,1) \times Y^{*}} \phi\left|\partial_{x} u \nabla_{y} v\right|^{p-2} \partial_{x} u \nabla_{y} v \nabla_{y} \varphi d x d Y=0, \quad \forall \varphi \in W_{\#}^{1, p}\left(Y^{*}\right) / \mathbb{R} .
$$

Thus, from the density of tensor product $C_{0}^{\infty}(0,1) \otimes\left(W_{\#}^{1, p}\left(Y^{*}\right) / \mathbb{R}\right)$, we get

$$
\int_{(0,1) \times Y^{*}}\left|\partial_{x} u \nabla_{y} v\right|^{p-2} \partial_{x} u \nabla_{y} v \nabla_{y} \psi d x d Y=0, \quad \forall \psi \in L^{p}\left((0,1) ; W_{\#}^{1, p}\left(Y^{*}\right) / \mathbb{R}\right) .
$$

Hence, we can conclude (16) from equations $(32)$ and (33). Moreover, we can rewrite $(19)$ as

$$
\int_{(0,1) \times Y^{*}}\left|\partial_{x} u \nabla_{y} v\right|^{p-2} \partial_{x} u \nabla_{y} v \nabla \varphi+|u|^{p-2} u \varphi d x d Y=\int_{(0,1) \times Y^{*}} \hat{f} \varphi d x d Y,
$$

which is equivalent to

$$
\int_{0}^{1} q\left|\partial_{x} u\right|^{p-2} \partial_{x} u \partial_{x} \varphi+|u|^{p-2} u \varphi d x=\int_{0}^{1} \bar{f} \varphi d x
$$

where $q$ is that one given by

$$
q=\int_{Y^{*}}\left|\nabla_{y} v\right|^{p-2} \partial_{y_{1}} v d Y
$$

and

$$
\bar{f}=\frac{1}{\left|Y^{*}\right|} \int_{Y^{*}} \hat{f} d Y
$$

We point out that $q>0$. Indeed, since $\left(\nabla_{y} v-(1,0)\right) \in W_{\#, 0}^{1, p}\left(Y^{*}\right)$, we get

$$
0<\int_{Y^{*}}\left|\nabla_{y} v\right|^{p} d Y=\int_{Y^{*}}\left|\nabla_{y} v\right|^{p-2} \nabla_{y} v\left[(1,0)+\nabla_{y} v-(1,0)\right] d Y=\int_{Y^{*}}\left|\nabla_{y} v\right|^{p-2} \partial_{y_{1}} v d Y .
$$

Finally, problem 13 is well posed, we conclude the proof noting that $T_{\varepsilon} u_{\varepsilon}$ is a convergent sequence.

In the proof of Theorem 3.1, we have already obtained a corrector result. The corrector function is given by $W_{\varepsilon}$. Indeed, according to [9], since we already have, by Propositions 2.13 and 2.11 .

$$
\left\|u_{\varepsilon}-u\right\| \|_{L^{p}\left(R^{\varepsilon}\right)} \rightarrow 0, \quad \text { as } \varepsilon \rightarrow 0
$$

we just need to construct the corrector to the term $\nabla u_{\varepsilon}$. We have the following result.

Corollary 3.1.1. The function $W_{\varepsilon}$ defined in 22 satisfies

$$
\left\|T_{\varepsilon} \nabla u_{\varepsilon}-T_{\varepsilon} W_{\varepsilon}\right\|_{L^{p}\left((0,1) \times Y^{*}\right)} \rightarrow 0 .
$$

Proof. The proof follows from (29), when $p \geq 2$, and (30), when $1<p \leq 2$.

Remark 3.1. From Corollary 3.1.1 and Proposition 2.17 of [6], we can conclude that

$$
\left\|\mid \nabla u_{\varepsilon}-W_{\varepsilon}\right\|_{L^{p}\left(R^{\varepsilon}\right)} \rightarrow 0, \quad \text { as } \varepsilon \rightarrow 0 .
$$

Consequently, due to 22), we obtain that the function

$$
\nabla_{y} u_{1}\left(x, \frac{x}{\varepsilon}, \frac{y}{\varepsilon}\right), \quad(x, y) \in R^{\varepsilon},
$$

works as a corrector function to $\nabla u_{\varepsilon}$ in $L^{p}\left(R^{\varepsilon}\right)$ with the norm $\| \cdot|| \mid$ since it allows strong convergence. 


\section{Weak oscillation case: $\alpha<1$.}

Now, let us consider $\alpha<1$. Here, we deal with the oscillatory thin domain $R^{\varepsilon}$ given by

$$
R^{\varepsilon}=\left\{(x, y) \in \mathbb{R}^{2}: 0<x<1,0<y<\varepsilon g\left(\frac{x}{\varepsilon^{\alpha}}\right)\right\}
$$

with $g$ satisfying hypothesis $\left(\mathbf{H}_{\mathbf{g}}\right)$ at Section 2 .

In order to obtain the homogenized equation, we will proceed as in the previous case $\alpha=1$, Section 3. We show the following result.

Theorem 4.1. Let $u_{\varepsilon}$ be the solution of problem (2) with $f^{\varepsilon}$ satisfying $\left\|\left|f^{\varepsilon}\right|\right\|_{L^{p^{\prime}\left(R^{\varepsilon}\right)}} \leq c$ for some positive constant independent of $\varepsilon>0$. Suppose also that

$$
T_{\varepsilon} f^{\varepsilon} \rightarrow \hat{f} \text { weakly in } L^{p^{\prime}}\left((0,1) \times Y^{*}\right) .
$$

Then, there exists $\left(u, u_{1}\right) \in W^{1, p}(0,1) \times L^{p}\left((0,1) ; W_{\#}^{1, p}\left(Y^{*}\right)\right)$ with $\partial_{y_{2}} u_{1}=0$ such that

$$
\begin{array}{r}
T_{\varepsilon} u_{\varepsilon} \rightarrow u \text { weakly in } L^{p}\left((0,1) ; W^{1, p}\left(Y^{*}\right)\right) \\
T_{\varepsilon} \partial_{x} u_{\varepsilon} \rightarrow \partial_{x} u+\partial_{y_{1}} u_{1} \text { weakly in } L^{p}\left((0,1) \times Y^{*}\right)
\end{array}
$$

where $u$ is the unique solution of

$$
\int_{0}^{1}\left[\frac{1}{\langle g\rangle_{(0, L)}\left\langle 1 / g^{p^{\prime}-1}\right\rangle_{(0, L)}^{p-1}}\left|\partial_{x} u\right|^{p-2} \partial_{x} u \partial_{x} \varphi+|u|^{p-2} u \varphi\right] d x=\int_{0}^{1} \bar{f} \varphi d x, \quad \forall \varphi \in W^{1, p}(0,1),
$$

and

$$
\bar{f}=\frac{1}{\left|Y^{*}\right|} \int_{Y^{*}} \hat{f} d y_{1} d y_{2}
$$

Proof. From Proposition 2.13 and Theorem 2.12 , there exist $u \in W^{1, p}(0,1)$ and $u_{1} \in L^{p}\left((0,1) ; W_{\#}^{1, p}\left(Y^{*}\right)\right)$ with $\partial_{y_{2}} u_{1}=0$ such that, up to subsequences,

$$
\begin{array}{r}
T_{\varepsilon} u_{\varepsilon} \rightarrow u \text { weakly in } L^{p}\left((0,1) ; W^{1, p}\left(Y^{*}\right)\right), \\
T_{\varepsilon}\left(\left|u_{\varepsilon}\right|^{p-2} u_{\varepsilon}\right) \rightarrow|u|^{p-2} u \text { strongly in } L^{p}\left((0,1) \times Y^{*}\right), \\
T_{\varepsilon} \partial_{x} u_{\varepsilon} \rightarrow \partial_{x} u+\partial_{y_{1}} u_{1} \text { weakly in } L^{p}\left((0,1) \times Y^{*}\right), \\
T_{\varepsilon}\left(\left|\nabla u_{\varepsilon}\right|^{p-2} \nabla u_{\varepsilon}\right) \rightarrow a_{0} \text { weakly in } L^{p^{\prime}}\left((0,1) \times Y^{*}\right)^{2} .
\end{array}
$$

We rewrite (3) and obtain as in (17) that

$$
\begin{aligned}
& \int_{\left(0,1 \times Y^{*}\right)} T_{\varepsilon}\left(\left|\nabla u_{\varepsilon}\right|^{p-2} \nabla u_{\varepsilon}\right) T_{\varepsilon} \nabla \varphi d x d y_{1} d y_{2}+\frac{L}{\varepsilon} \int_{R_{1}^{\varepsilon}}\left|\nabla u_{\varepsilon}\right|^{p-2} \nabla u_{\varepsilon} \nabla \varphi d x d y \\
& +\int_{\left(0,1 \times Y^{*}\right)} T_{\varepsilon}\left(\left|u_{\varepsilon}\right|^{p-2} u_{\varepsilon}\right) T_{\varepsilon} \varphi d x d y_{1} d y_{2}+\frac{L}{\varepsilon} \int_{R_{1}^{\varepsilon}}\left|u_{\varepsilon}\right|^{p-2} u_{\varepsilon} \varphi d x d y \\
& =\int_{\left(0,1 \times Y^{*}\right)} T_{\varepsilon} f^{\varepsilon} T_{\varepsilon} \varphi d x d y_{1} d y_{2}+\frac{L}{\varepsilon} \int_{R_{1}^{\varepsilon}} f^{\varepsilon} \varphi d x d y, \quad \forall \varphi \in W^{1, p}(0,1) .
\end{aligned}
$$

Hence, we can pass to the limit to get

$$
\begin{aligned}
\int_{(0,1) \times Y^{*}} & a_{0} \nabla \varphi d x d y_{1} d y_{2}+\int_{(0,1) \times Y^{*}}|u|^{p-2} u \varphi d x d y_{1} d y_{2} \\
= & \int_{(0,1) \times Y^{*}} \hat{f} \varphi d x d y_{1} d y_{2} .
\end{aligned}
$$

Now, let $\phi \in C_{0}^{\infty}(0,1)$ and $\psi \in W_{\#}^{1, p}\left(Y^{*}\right)$ with $\partial_{y_{2}} \psi=0$ (that is, assume $\psi\left(y_{1}, y_{2}\right)=\psi\left(y_{1}\right)$ ). Define the sequence

$$
v_{\varepsilon}(x, y)=\varepsilon^{\alpha} \phi(x) \psi\left(\frac{x}{\varepsilon^{\alpha}}\right) .
$$


Then, we have that

$$
\begin{aligned}
& T_{\varepsilon} v_{\varepsilon} \rightarrow 0 \text { strongly in } L^{p}\left((0,1) \times Y^{*}\right), \\
& T_{\varepsilon}\left(\partial_{x} v_{\varepsilon}\right) \rightarrow \phi \partial_{y_{1}} \psi \text { strongly in } L^{p}\left((0,1) \times Y^{*}\right), \\
& T_{\varepsilon}\left(\partial_{y} v_{\varepsilon}\right)=0
\end{aligned}
$$

Next, taking $v_{\varepsilon}$ as a test function in (35), we have, as $\varepsilon \rightarrow 0$, that

$$
\int_{(0,1) \times Y^{*}} a_{0} \cdot(1,0) \phi(x) \partial_{y_{1}} \psi d x d y_{1} d y_{2}=0 .
$$

Hence, by the density of the tensor product $C_{0}^{\infty}(0,1) \otimes W_{\#}^{1, p}\left(Y^{*}\right)$ in $L^{p}\left((0,1) ; W_{\#}^{1, p}\left(Y^{*}\right)\right)$, we obtain

$$
\int_{(0,1) \times Y^{*}} a_{0} \cdot(1,0) \partial_{y_{1}} \psi d x d y_{1} d y_{2}=0, \quad \forall \psi \in L^{p}\left((0,1) ; W_{\#}^{1, p}\left(Y^{*}\right)\right),
$$

for all $\psi \in L^{p}\left((0,1) ; W_{\#}^{1, p}\left(Y^{*}\right)\right)$ such that $\partial_{y_{2}} \psi=0$.

Now, we identify $a_{0}$. We argue as in the previous section. We take the limit functions $u \in W^{1, p}(0,1)$ and $u_{1} \in L^{p}\left((0,1) ; W_{\#}^{1, p}\left(Y^{*}\right)\right)$, we extend $\partial_{y_{1}} u_{1}$ in $y_{1}$-direction and define the sequence

$$
W_{\varepsilon}(x)=\left(\partial_{x} u(x)+\partial_{y_{1}} u_{1}\left(x, \frac{x}{\varepsilon^{\alpha}}\right), 0\right) .
$$

Notice that by Proposition 2.8 we have

$$
\begin{aligned}
& T_{\varepsilon} W_{\varepsilon} \rightarrow\left(\partial_{x} u+\partial_{y_{1}} u_{1}, 0\right) \text { in } L^{p}\left((0,1) \times Y^{*}\right)^{2}, \\
& T_{\varepsilon}\left(\left|W_{\varepsilon}\right|^{p-2} W_{\varepsilon}\right) \rightarrow\left|\partial_{x} u+\partial_{y_{1}} u_{1}\right|^{p-2}\left[\left(\partial_{x} u+\partial_{y_{1}} u_{1}, 0\right)\right] \text { in } L^{p^{\prime}}\left((0,1) \times Y^{*}\right)^{2},
\end{aligned}
$$

and then, $T_{\varepsilon}\left(\nabla u_{\varepsilon}-W_{\varepsilon}\right) \rightarrow 0$ weakly in $L^{p}\left((0,1) \times Y^{*}\right)$.

Analogously to the resonant case, we have as in (28) and (31) that

$$
\begin{aligned}
& \int_{(0,1) \times Y^{*}} T_{\varepsilon}\left[\left|\nabla u_{\varepsilon}\right|^{p-2} \nabla u_{\varepsilon}-\left|W_{\varepsilon}\right|^{p-2} W_{\varepsilon}\right] T_{\varepsilon}\left(\nabla u_{\varepsilon}-W_{\varepsilon}\right) d x d y_{1} d y_{2} \rightarrow 0, \\
& \int_{(0,1) \times Y^{*}} T_{\varepsilon}\left[\left|\nabla u_{\varepsilon}\right|^{p-2} \nabla u_{\varepsilon}-\left|W_{\varepsilon}\right|^{p-2} W_{\varepsilon}\right] \varphi d x d y_{1} d y_{2} \rightarrow 0,
\end{aligned}
$$

for any $\varphi \in C_{0}^{\infty}\left((0,1) \times Y^{*}\right)^{2}$. Therefore,

$$
T_{\varepsilon}\left(\left|\nabla u_{\varepsilon}\right|^{p-2} \nabla u_{\varepsilon}\right) \rightarrow\left|\partial_{x} u+\partial_{y_{1}} u_{1}\right|^{p-2}\left(\partial_{x} u+\partial_{y_{1}} u_{1}, 0\right), \quad \text { weakly in } L^{p^{\prime}}\left((0,1) \times Y^{*}\right)^{2}
$$

and then,

$$
a_{0}=\left|\partial_{x} u+\partial_{y_{1}} u_{1}\right|^{p-2}\left(\partial_{x} u+\partial_{y_{1}} u_{1}, 0\right) \text { a.e }(0,1) \times Y^{*} .
$$

Thus, due to (36), we get

$$
\begin{aligned}
\int_{(0,1) \times Y^{*}} & \left(\hat{f}-|u|^{p-2} u\right) \varphi d x d y_{1} d y_{2} \\
& -\int_{(0,1) \times Y^{*}}\left|\partial_{x} u+\partial_{y_{1}} u_{1}\right|^{p-2}\left(\partial_{x} u+\partial_{y_{1}} u_{1}\right) \partial_{x} \varphi d x d y_{1} d y_{2}=0
\end{aligned}
$$

for all $\varphi \in W^{1, p}(0,1)$.

Since $u \in W^{1, p}(0,1), \partial_{y_{2}} \psi=0$ and $\partial_{y_{2}} u_{1}=0$, we can take $\psi \in L^{p}\left((0,1) ; W_{\#}^{1, p}(0, L)\right)$, and then, using (39), we can rewrite (37) as

$$
\int_{(0,1) \times(0, L)}\left|\partial_{x} u+\partial_{y_{1}} u_{1}\right|^{p-2}\left(\partial_{x} u+\partial_{y_{1}} u_{1}\right) \partial_{y_{1}} \psi g\left(y_{1}\right) d x d y_{1}=0
$$


for all $\psi \in L^{p}\left((0,1) ; W_{\#}^{1, p}(0, L)\right)$.

Hence, treating $x$ as a parameter in the above equation we have that there exists a function $T$ depending only on $x$ such that

$$
T(x)=\left|\partial_{x} u+\partial_{y_{1}} u_{1}\right|^{p-2}\left(\partial_{x} u+\partial_{y_{1}} u_{1}\right) g\left(y_{1}\right) \text { a.e }(0, L) .
$$

By Corollary 2.1.1, the composition of $a_{p}$ and $a_{p^{\prime}}$ gives the identity. Thus, we can rewrite the above equality as

$$
\partial_{x} u+\partial_{y_{1}} u_{1}=\left|\frac{T}{g}\right|^{p^{\prime}-2} \frac{T}{g} .
$$

Using the periodicity of $u_{1}$, we get

$$
0=\int_{0}^{L} \partial_{y_{1}} u_{1} d y_{1}=\int_{0}^{L}\left|\frac{T}{g}\right|^{p^{\prime}-2} \frac{T}{g}-\partial_{x} u d y_{1},
$$

which means that

$$
|T|^{p^{\prime}-2} T=\partial_{x} u /\left\langle\frac{1}{|g|^{p^{\prime}-2} g}\right\rangle_{(0, L)}
$$

Putting this together with 41, one can get

$$
\partial_{y_{1}} u_{1}=\partial_{x} u \frac{1}{|g|^{p^{\prime}-2} g\left\langle 1 /|g|^{p^{\prime}-2} g\right\rangle_{(0, L)}}-\partial_{x} u .
$$

Thus, using the above equality and the fact that $g>0$, we can rewrite 40 as

$$
\int_{0}^{1} \frac{1}{\langle g\rangle_{(0, L)}\left\langle 1 / g^{p^{\prime}-1}\right\rangle_{(0, L)}^{p-1}}\left|\partial_{x} u\right|^{p-2} \partial_{x} u \partial_{x} \varphi+|u|^{p-2} u \varphi d x=\int_{0}^{1} \bar{f} \varphi d x
$$

for all $\varphi \in W^{1, p}(0,1)$, where

$$
\bar{f}=\frac{1}{\left|Y^{*}\right|} \int_{Y^{*}} \hat{f} d y_{1} d y_{2}
$$

As in Corollary 3.1.1, we get the following corrector result to 22 for $\alpha \in(0,1)$ :

Corollary 4.1.1. The function $W_{\varepsilon}$ defined in (38) satisfies

$$
\left\|T_{\varepsilon} \nabla u_{\varepsilon}-T_{\varepsilon} W_{\varepsilon}\right\|_{L^{p}\left((0,1) \times Y^{*}\right)} \rightarrow 0 .
$$

Remark 4.1. From Corollary 4.1.1 and [6, Proposition 2.17], we have that

$$
\left\|\mid \nabla u_{\varepsilon}-W_{\varepsilon}\right\|_{L^{p}\left(R^{\varepsilon}\right)} \rightarrow 0 .
$$

\section{The strong oscillation case: $\alpha>1$.}

In this section we analyze the behavior of the solutions of $(2)$ as the upper boundary of the thin domains presents a very high oscillatory boundary. Then, the thin domain is defined as follows

$$
R^{\varepsilon}=\left\{(x, y) \in \mathbb{R}^{2}: 0<x<1,0<y<\varepsilon g\left(\frac{x}{\varepsilon^{\alpha}}\right)\right\} .
$$

where $\alpha>1$ and $g$ satisfies the hypothesis $\left(\mathbf{H}_{\mathbf{g}}\right)$.

We would like to point out that even though we use the unfolding operator to get the homogenized limit problem, the approach is different to the two previous cases. The roughness is so strong that we 
can not obtain a compactness theorem as in the previous results Theorems 2.12 and 2.12 . To overcome this difficulty we will divide the thin domain in two thin parts:

$$
\begin{aligned}
& R_{+}^{\varepsilon}=\left\{(x, y) \in \mathbb{R}^{2}: x \in(0,1), \varepsilon g_{0}<y<\varepsilon g\left(x / \varepsilon^{\alpha}\right)\right\}, \\
& R_{-}^{\varepsilon}=\left\{(x, y) \in \mathbb{R}^{2}: x \in(0,1), 0<y<g_{0}\right\} .
\end{aligned}
$$

Notice that

$$
R^{\varepsilon}=\operatorname{Int}\left(\overline{R_{+}^{\varepsilon}} \bigcup \overline{R_{-}^{\varepsilon}}\right) .
$$

Moreover, let us introduce the following sets independent of $\varepsilon$

$$
\begin{aligned}
& Y^{*}=\left\{\left(y_{1}, y_{2}\right) \in \mathbb{R}^{2}: 0<y_{1}<L, 0<y_{2}<g\left(y_{1}\right)\right\}, \\
& Y_{+}^{*}=\left\{\left(y_{1}, y_{2}\right) \in \mathbb{R}^{2}: 0<y_{1}<L, g_{0}<y_{2}<g\left(y_{1}\right)\right\}, \\
& R_{-}=\left\{(x, y) \in \mathbb{R}^{2}: x \in(0,1), 0<y<g_{0}\right\}, \\
& R_{+}=\left\{(x, y) \in \mathbb{R}^{2}: x \in(0,1), g_{0}<y<g_{1}\right\} .
\end{aligned}
$$

Remark 5.1. Notice that the reference cell for the unfolding operator restricted to the oscillating part, $Y_{+}^{*}$, may be disconnected since $g_{0}=\min _{x \in \mathbb{R}} g(x)$.

We first introduce an operator which allows us to rescale $R_{-}^{\varepsilon}$ in order to work over a fixed domain.

Definition 5.1. Let $\varphi \in L^{p}\left(R_{-}^{\varepsilon}\right)$. The rescaling operator $\Pi_{\varepsilon}: L^{p}\left(R_{-}^{\varepsilon}\right) \rightarrow L^{p}\left(R_{-}\right)$is defined by

$$
\Pi_{\varepsilon}(\varphi)(x, y)=\varphi(x, \varepsilon y), \forall(x, y) \in R_{-} .
$$

Proposition 5.2. The rescaling operator satisfies the following properties:

1. Let $\varphi \in L^{1}\left(R_{-}^{\varepsilon}\right)$. Then

$$
\int_{R_{-}} \Pi_{\varepsilon}(\varphi)(x, y) d x d y=\frac{1}{\varepsilon} \int_{R_{-}^{\varepsilon}} \varphi(x, y) d x d y
$$

2. $\Pi_{\varepsilon}$ is linear and continuous from $L^{p}\left(R_{-}^{\varepsilon}\right)$ to $L^{p}\left(R_{-}\right), 1 \leq p \leq \infty$. In addition, the following relationship existis between their norms:

$$
\begin{gathered}
\left\|\Pi_{\varepsilon}(\cdot)\right\|_{L^{p}\left(R_{-}\right)}=\left|\|\cdot \mid\|_{L^{p}\left(R_{-}^{\varepsilon}\right)},\right. \\
\left\|\Pi_{\varepsilon}(\cdot)\right\|_{L^{\infty}\left(R_{-}\right)}=\||| \cdot||_{L^{\infty}\left(R_{-}^{\varepsilon}\right)} .
\end{gathered}
$$

3. For $\varphi \in W^{1, p}\left(R_{-}^{\varepsilon}\right), 1 \leq p \leq \infty$, we have

$$
\partial_{x} \Pi_{\varepsilon} \varphi=\Pi_{\varepsilon} \partial_{x} \varphi, \quad \partial_{y} \Pi_{\varepsilon} \varphi=\varepsilon \Pi_{\varepsilon} \partial_{y} \varphi
$$

4. Let $\phi \in L^{p}(0,1), 1 \leq p \leq \infty$. Then, considering $\phi$ as a function defined in $R_{-}^{\varepsilon}$, we have $\Pi_{\varepsilon} \phi=\phi$. Proof. The proof follows directly from the definition.

Our homogenization result is the following one.

Theorem 5.3. Let $u_{\varepsilon}$ be the solution of the problem (2) with $f^{\varepsilon} \in L^{p^{\prime}}\left(R^{\varepsilon}\right)$ and $\left\|f^{\varepsilon}\right\|_{L^{p^{\prime}\left(R^{\varepsilon}\right)}} \leq c$, for some $c>0$ independent of $\varepsilon$. Suppose the function

$$
\hat{f}^{\varepsilon}(x)=\frac{1}{\varepsilon} \int_{0}^{\varepsilon g\left(\frac{x}{\varepsilon^{\alpha}}\right)} f(x, y) d y
$$

satisfies

$$
\hat{f}^{\varepsilon} \rightarrow \hat{f} \text { weakly in } L^{p^{\prime}}(0,1) .
$$


Then, there exists a unique $u \in W^{1, p}(0,1)$ such that

$$
\begin{gathered}
T_{\varepsilon} u_{\varepsilon} \rightarrow u \text { weakly in } L^{p}\left((0,1) ; W^{1, p}\left(Y^{*}\right)\right), \\
\text { and } \quad\left\|\left|u_{\varepsilon}-u\right|\right\|_{L^{p}\left(R^{\varepsilon}\right)} \rightarrow 0,
\end{gathered}
$$

as $\varepsilon \rightarrow 0$.

Moreover, $u$ is the unique solution of the one dimensional p-Laplacian problem

$$
\begin{gathered}
\int_{0}^{1}\left(\frac{g_{0}}{\langle g\rangle_{(0, L)}}\left|\partial_{x} u\right|^{p-2} \partial_{x} u \partial_{x} \varphi+|u|^{p-2} u \varphi\right) d x=\int_{0}^{1} \bar{f} \varphi d x \\
\bar{f}=\frac{\hat{f}}{\langle g\rangle_{(0, L)}} .
\end{gathered}
$$

Proof. Throughout this proof, we denote by $T_{\varepsilon}$ the unfolding operator associated to the cell $Y^{*}$, $T_{\varepsilon}: L^{p}\left(R^{\varepsilon}\right) \rightarrow L^{p}\left((0,1) \times Y^{*}\right)$ and by $T_{\varepsilon}^{+}$the unfolding operator associated to the cell $Y_{+}^{*}, T_{\varepsilon}^{+}$: $L^{p}\left(R_{+}^{\varepsilon}\right) \rightarrow L^{p}\left((0,1) \times Y_{+}^{*}\right)$.

By Proposition 2.13, we have uniform bound of solutions. Therefore, from Proposition 2.11, there exists $u \in W^{1, p}(0,1)$ and $a_{0} \in L^{p^{\prime}}\left((0,1) \times Y^{*}\right)^{2}$ such that, up to subsequences,

$$
\begin{aligned}
& \lim _{\varepsilon \rightarrow 0}\left|\| u_{\varepsilon}-u\right|||_{L^{p}\left(R^{\varepsilon}\right)}=0 \\
& T_{\varepsilon} u_{\varepsilon} \rightarrow u, \text { weakly in } L^{p}\left((0,1) ; W^{1, p}\left(Y^{*}\right)\right) \\
& T_{\varepsilon} u_{\varepsilon} \rightarrow u, \text { strongly in } L^{p}\left((0,1) \times Y^{*}\right), \\
& T_{\varepsilon}\left(\left|\nabla u_{\varepsilon}\right|^{p-2} \nabla u_{\varepsilon}\right) \rightarrow a_{0} \text { weakly in } L^{p^{\prime}}\left((0,1) \times Y^{*}\right)^{2} .
\end{aligned}
$$

In order to simplify the notations, we denote the following restrictions by

$$
u_{\varepsilon}^{+}=\left.u_{\varepsilon}\right|_{R_{+}^{\varepsilon}} \quad \text { and } \quad u_{\varepsilon}^{-}=\left.u_{\varepsilon}\right|_{R_{-}^{\varepsilon}} .
$$

From Proposition 2.13, we have that $T_{\varepsilon}\left(\left|\nabla u_{\varepsilon}^{+}\right|^{p-2} \nabla u_{\varepsilon}^{+}\right)$is uniformly bounded. Therefore, there exists $u_{1} \in L^{p}\left((0,1) \times Y^{*}\right)^{2}$ such that, up to subsequences,

$$
T_{\varepsilon}\left(\left|\nabla u_{\varepsilon}^{+}\right|^{p-2} \partial_{x} u_{\varepsilon}^{+}\right) \rightarrow u_{1} \text { weakly in } L^{p}\left((0,1) \times Y^{*}\right) .
$$

We show that $u_{1}\left(x, y_{1}, y_{2}\right)=0$ a.e. in $(0,1) \times Y_{+}^{*}$. To do this, we proceed as in [6, Theorem 5.3] using the suitable test functions there introduced.

Since $g_{0}=\min _{x \in \mathbb{R}} g(x)$ and $g$ is $L$-periodic, there is, at least, a point $y_{0}$ such that $g\left(y_{0}\right)=g_{0}$. Furthermore,

$$
\left\{\left(y_{0}, y_{2}\right) \in \mathbb{R}^{2}: y_{2} \in\left(g_{0}, g_{1}\right)\right\} \cap Y_{+}^{*}=\emptyset .
$$

We analyze two cases: $y_{0}>0$ and $y_{0}=0$.

First, suppose $y_{0}>0$. Then, for any $\phi \in C_{0}^{\infty}\left(0, y_{0}\right)$ we define the following function:

$$
\psi\left(y_{1}\right)=\left\{\begin{array}{l}
\int_{0}^{y_{1}} \phi(t) d t, \text { if } 0 \leq y_{1}<y_{0} \\
0, \text { if } y_{0}<y_{1}<L .
\end{array}\right.
$$

Notice that $\psi$ can be extended by $L$-periodicity and $\psi \in C^{\infty}\left[0, y_{0}\right) \cup C^{\infty}\left(y_{0}, L\right)$. Then, we consider the test function

$$
\varphi^{\varepsilon}(x, y)=\varepsilon^{\alpha} \tilde{\varphi}\left(x, \frac{y}{\varepsilon}\right) \psi\left(\left\{\frac{x}{\varepsilon^{\alpha}}\right\}\right), \quad(x, y) \in R^{\varepsilon},
$$

where $\varphi \in C_{0}^{\infty}\left(R_{+}\right), \psi$ is defined in (45) and $\sim$ is the extension by zero. Using (44) and the definition of (45), we have that $\varphi^{\varepsilon}$ is continuous in $R^{\varepsilon}$ for each $\varepsilon$.

Now, we apply the unfolding operator to the restricition of $\varphi^{\varepsilon}$ to the thin domain $R_{+}^{\varepsilon}$ :

$$
T_{\varepsilon}^{+}\left(\varphi^{\varepsilon}\right)\left(x, y_{1}, y_{2}\right)=\left\{\begin{array}{l}
\varepsilon^{\alpha} \varphi\left(\varepsilon^{\alpha}\left[\frac{x}{\varepsilon^{\alpha}}\right] L+\varepsilon^{\alpha} y_{1}, y_{2}\right) \psi\left(y_{1}\right) \text { for }\left(x, y_{1}, y_{2}\right) \in I_{\varepsilon} \times Y_{+}^{*} \\
0 \text { for }\left(x, y_{1}, y_{2}\right) \in \Lambda_{\varepsilon} \times Y_{+}^{*}
\end{array}\right.
$$


By Proposition 2.3, we have

$$
\begin{aligned}
T_{\varepsilon}^{+}\left(\partial_{x} \varphi^{\varepsilon}\right)=\frac{1}{\varepsilon^{\alpha}} \partial_{y_{1}} T_{\varepsilon}^{+} \varphi^{\varepsilon} & =\varepsilon^{\alpha} T_{\varepsilon}^{+}\left(\partial_{x} \varphi\right) \psi\left(y_{1}\right)+\psi^{\prime}\left(y_{1}\right) T_{\varepsilon}^{+}(\varphi(\cdot, \cdot / \varepsilon)), \\
\text { and } T_{\varepsilon}^{+}\left(\partial_{y} \varphi^{\varepsilon}\right) & =\frac{1}{\varepsilon} \partial_{y_{2}} T_{\varepsilon}^{+} \varphi^{\varepsilon}=\varepsilon^{\alpha-1} T_{\varepsilon}^{+}\left(\partial_{y} \varphi(\cdot, \cdot / \varepsilon)\right) \psi\left(y_{1}\right) .
\end{aligned}
$$

Since $\alpha>1$, we obtain from Proposition 2.8 that

$$
\begin{gathered}
T_{\varepsilon}^{+} \varphi^{\varepsilon} \rightarrow 0 \text { strongly in } L^{p}\left((0,1) \times Y_{+}^{*}\right), \\
T_{\varepsilon}^{+}\left(\partial_{x} \varphi^{\varepsilon}\right) \rightarrow \psi^{\prime}\left(y_{1}\right) \varphi\left(x, y_{2}\right) \text { strongly in } L^{p}\left((0,1) \times Y_{+}^{*}\right), \\
T_{\varepsilon}^{+}\left(\partial_{y} \varphi^{\varepsilon}\right) \rightarrow 0 \text { strongly in } L^{p}\left((0,1) \times Y_{+}^{*}\right) .
\end{gathered}
$$

We use the fact that $\varphi^{\varepsilon}$ annihilates in $R_{-}^{\varepsilon}$ to take it as a test function in (3) to obtain

$$
\int_{R_{+}^{\varepsilon}}\left|\nabla u_{\varepsilon}\right|^{p-2} \nabla u_{\varepsilon} \nabla \varphi^{\varepsilon}+\left|u_{\varepsilon}\right|^{p-2} u_{\varepsilon} \varphi^{\varepsilon} d x d y=\int_{R_{+}^{\varepsilon}} f^{\varepsilon} \varphi^{\varepsilon} d x d y
$$

Thus, from 43) and 460), we get

$$
\int_{(0,1) \times Y_{+}^{*}} u_{1} \psi^{\prime}\left(y_{1}\right) \varphi\left(x, y_{2}\right) d x d y_{1} d y_{2}=0 .
$$

That is,

$$
\int_{(0,1) \times\left(g_{0}, g_{1}\right)} \varphi\left(x, y_{2}\right)\left(\int_{0}^{L} \tilde{u}_{1} \psi^{\prime} d y_{1}\right) d y_{2} d x=0, \quad \forall \varphi \in C_{0}^{\infty}\left(R_{+}\right) .
$$

Thus,

$$
\int_{0}^{L} \tilde{u}_{1}\left(x, y_{1}, y\right) \psi^{\prime} d y_{1}=0 \quad \text { a.e. }(x, y) \in R_{+},
$$

and then, from 45), we have

$$
\int_{0}^{y_{0}} \tilde{u}_{1}\left(x, y_{1}, y\right) \phi\left(y_{1}\right) d y_{1}=0 \quad \text { a.e. }(x, y) \in R_{+} \text {and } \forall \phi \in C_{0}^{\infty}\left(0, y_{0}\right) .
$$

Hence, we have

$$
\tilde{u}_{1}\left(x, y_{1}, y_{2}\right)=0 \text { a.e. }\left(x, y_{1}, y_{2}\right) \in(0,1) \times\left(0, y_{0}\right) \times\left(g_{0}, g_{1}\right) .
$$

Now, repeating the arguments to $\psi$ defined as

$$
\psi\left(y_{1}\right)=\left\{\begin{array}{l}
0, \text { if } 0 \leq x<y_{0}, \\
\int_{y_{0}}^{y_{1}} \phi(t) d t-\int_{y_{0}}^{L} \phi(t) d t, \text { if } y_{0}<x<L,
\end{array}\right.
$$

with $\phi \in C_{0}^{\infty}\left(y_{0}, L\right)$, we get

$$
\tilde{u}_{1}\left(x, y_{1}, y_{2}\right)=0 \text { a.e. }\left(x, y_{1}, y_{2}\right) \in(0,1) \times\left(y_{0}, L\right) \times\left(g_{0}, g_{1}\right) .
$$

Therefore,

$$
u_{1}\left(x, y_{1}, y_{2}\right)=0 \text { a.e. }\left(x, y_{1}, y_{2}\right) \in(0,1) \times Y_{+}^{*} .
$$

Finally, for $y_{0}=0$, set

$$
\psi\left(y_{1}\right)=\int_{0}^{y_{1}} \phi(t) d t, \text { with } 0<y_{1}<L
$$

for any $\phi \in C_{0}^{\infty}(0, L)$. In this case, we have

$$
\left\{\left(0, y_{2}\right): y_{2} \in\left(g_{0}, g_{1}\right)\right\} \cap Y_{+}^{*}=\emptyset \text {, and }\left\{\left(L, y_{2}\right): y_{2} \in\left(g_{0}, g_{1}\right)\right\} \cap Y_{+}^{*}=\emptyset .
$$


Then, the sequence

$$
\varphi^{\varepsilon}(x, y)=\varepsilon^{\alpha} \tilde{\varphi}\left(x, \frac{y}{\varepsilon}\right) \psi\left(\left\{\frac{x}{\varepsilon^{\alpha}}\right\}\right), \quad(x, y) \in R^{\varepsilon},
$$

is well defined since $\varphi \in C_{0}^{\infty}\left(R_{+}\right)$.

Thus, using the same arguments as before, we get that $u_{1}=0$ a.e. $\left(x, y_{1}, y_{2}\right) \in(0,1) \times Y_{+}^{*}$. Therefore,

$$
T_{\varepsilon}\left(\left|\nabla u_{\varepsilon}^{+}\right|^{p-2} \partial_{x} u_{\varepsilon}^{+}\right) \rightarrow 0 \quad \text { weakly in } L^{p}\left((0,1) \times Y_{+}^{*}\right),
$$

and then,

$$
a_{0} \cdot(1,0)=u_{1}=0 \quad \text { a.e. }\left(x, y_{1}, y_{2}\right) \in(0,1) \times Y_{+}^{*} .
$$

For the function $u_{\varepsilon}^{-}$, due to Propositions 2.13 and 5.2 , there exists $u^{-} \in W^{1, p}(0,1)$ such that, up to subsequences,

$$
\begin{aligned}
& \Pi_{\varepsilon} u_{\varepsilon}^{-} \rightarrow u^{-} \text {weakly in } W^{1, p}\left(R_{-}\right), \\
& \Pi_{\varepsilon} \partial_{x} u_{\varepsilon}^{-} \rightarrow \partial_{x} u^{-} \text {weakly in } L^{p}\left(R_{-}\right), \\
& \Pi_{\varepsilon} \partial_{y} u_{\varepsilon}^{-} \rightarrow 0 \text { weakly in } L^{p}\left(R_{-}\right) .
\end{aligned}
$$

Furthermore, from items (2) and (4) of Proposition 5.2, we have

$$
\left\|\Pi_{\varepsilon} u_{\varepsilon}^{-}-u\right\|_{L^{p}\left(R_{-}\right)}=\left\||| u_{\varepsilon}^{-}-u\right\|\left\|_{L^{p}\left(R_{-}^{\varepsilon}\right)} \leq\right\||| u_{\varepsilon}-u\|\|_{L^{p}\left(R^{\varepsilon}\right)} .
$$

Thus,

$$
\Pi_{\varepsilon} u_{\varepsilon}^{-} \rightarrow u \text { strongly in } L^{p}\left(R_{-}\right)
$$

which means that

$$
u(x)=u_{-}(x) \text { a.e. in }(0,1) .
$$

Next, we pass to the limit in $\Pi_{\varepsilon}\left(\left|\nabla u_{\varepsilon}^{-}\right|^{p-2} \nabla u_{\varepsilon}^{-}\right)$. We show that

$$
\Pi_{\varepsilon}\left(\left|\nabla u_{\varepsilon}^{-}\right|^{p-2} \nabla u_{\varepsilon}^{-}\right) \rightarrow\left|u^{\prime}\right|^{p-2}\left(u^{\prime}, 0\right) \text { in } L^{p}\left(R_{-}\right)^{2} .
$$

In order to do that, suppose that $a_{1}$ is the limit of $\Pi_{\varepsilon}\left(\left|\nabla u_{\varepsilon}^{-}\right|^{p-2} \nabla u_{\varepsilon}^{-}\right)$, up to subsequences. Again, we use monotonicity arguments. Let $w \in W^{1, p}(0,1)$.

Using $T_{\varepsilon}, T_{\varepsilon}^{+}$and the rescaling operator $\Pi_{\varepsilon}$ in the variational formulation (3), we obtain

$$
\begin{aligned}
& \frac{1}{L} \int_{(0,1) \times Y_{+}^{*}} T_{\varepsilon}^{+}\left(\left|\nabla u_{\varepsilon}^{+}\right|^{p-2} \nabla u_{\varepsilon}^{+}\right) T_{\varepsilon}^{+} \nabla \varphi d x d y_{1} d y_{2} \\
& +\frac{1}{\varepsilon} \int_{R_{1+}^{\varepsilon}}\left|\nabla u_{\varepsilon}^{+}\right|^{p-2} \nabla u_{\varepsilon}^{+} \nabla \varphi d x d y \\
& +\int_{R_{-}} \Pi_{\varepsilon}\left(\left|\nabla u_{\varepsilon}^{-}\right|^{p-2} \nabla u_{\varepsilon}^{-}\right) \Pi_{\varepsilon} \nabla \varphi d x d y \\
& +\frac{1}{L} \int_{(0,1) \times Y^{*}} T_{\varepsilon}\left(\left|u_{\varepsilon}\right|^{p-2} u_{\varepsilon}\right) T_{\varepsilon} \varphi d x d y_{1} d y_{2} \\
& +\frac{1}{\varepsilon} \int_{R_{1}^{\varepsilon}}\left|u_{\varepsilon}\right|^{p-2} u_{\varepsilon} \varphi d x d y \\
& =\frac{1}{\varepsilon} \int_{R^{\varepsilon}} f^{\varepsilon} \varphi d x d y .
\end{aligned}
$$

Hence, we can pass to the limit taking test functions $\varphi \in W^{1, p}(0,1)$ to obtain

$$
\int_{R_{-}} a_{1}\left(\varphi^{\prime}, 0\right) d x d y+\frac{1}{L} \int_{(0,1) \times Y^{*}}|u|^{p-2} u \varphi d x d y_{1} d y_{2}=\int_{0}^{1} \hat{f} \varphi d x
$$

Now, notice that

$$
0 \leq \frac{1}{L} \int_{(0,1) \times Y_{+}^{*}}\left|T_{\varepsilon}^{+} \nabla u_{\varepsilon}^{+}\right|^{p} d x d y_{1} d y_{2},
$$


also, due to 47, we have

$$
0=\lim _{\varepsilon \rightarrow 0}\left(\frac{1}{L} \int_{(0,1) \times Y_{+}^{*}}\left|T_{\varepsilon}^{+} \nabla u_{\varepsilon}^{+}\right|^{p-2} T_{\varepsilon}^{+} \nabla u_{\varepsilon}^{+}\left(T_{\varepsilon}^{+} u^{\prime}, 0\right) d x d y_{1} d y_{2}\right)
$$

since $T_{\varepsilon}^{+} u^{\prime} \rightarrow u^{\prime}$ strongly in $L^{p}\left((0,1) \times Y^{*}\right)$.

Using the monotonicity of $|\cdot|^{p-2}$, we have

$$
\begin{aligned}
0 \leq & \int_{R_{-}}\left(\Pi_{\varepsilon}\left(\left|\nabla u_{\varepsilon}^{-}\right|^{p-2} \nabla u_{\varepsilon}^{-}\right)-\Pi_{\varepsilon}\left(\left|u^{\prime}\right|^{p-2}\left(u^{\prime}, 0\right)\right)\right)\left(\Pi_{\varepsilon} \nabla u_{\varepsilon}^{-}-\Pi_{\varepsilon}\left(u^{\prime}, 0\right)\right) d x d y \\
\leq & \int_{R_{-}}\left(\Pi_{\varepsilon}\left(\left|\nabla u_{\varepsilon}^{-}\right|^{p-2} \nabla u_{\varepsilon}^{-}\right)-\Pi_{\varepsilon}\left(\left|u^{\prime}\right|^{p-2}\left(u^{\prime}, 0\right)\right)\right)\left(\Pi_{\varepsilon} \nabla u_{\varepsilon}^{-}-\Pi_{\varepsilon}\left(u^{\prime}, 0\right)\right) d x d y \\
& +\frac{1}{L} \int_{(0,1) \times Y_{+}^{*}}\left|T_{\varepsilon}^{+} \nabla u_{\varepsilon}^{+}\right|^{p} d x d y_{1} d y_{2}-\frac{1}{L} \int_{(0,1) \times Y_{+}^{*}}\left|T_{\varepsilon}^{+} \nabla u_{\varepsilon}^{+}\right|^{p-2} T_{\varepsilon}^{+} \nabla u_{\varepsilon}^{+}\left(T_{\varepsilon}^{+} u^{\prime}, 0\right) d x d y_{1} d y_{2} \\
& +\frac{1}{L} \int_{(0,1) \times Y_{+}^{*}}\left|T_{\varepsilon}^{+} \nabla u_{\varepsilon}^{+}\right|^{p-2} T_{\varepsilon}^{+} \nabla u_{\varepsilon}^{+}\left(T_{\varepsilon}^{+} u^{\prime}, 0\right) d x d y_{1} d y_{2}
\end{aligned}
$$

Now, we can pass to the limit in (49) using the variational formulation 48). Taking the test functions $\varphi=u_{\varepsilon}-u$ in (48), we have that

$$
\begin{gathered}
\frac{1}{L} \int_{(0,1) \times Y_{+}^{*}}\left|T_{\varepsilon}^{+} \nabla u_{\varepsilon}^{+}\right|^{p} d x d y_{1} d y_{2}-\frac{1}{L} \int_{(0,1) \times Y_{+}^{*}}\left|T_{\varepsilon}^{+} \nabla u_{\varepsilon}^{+}\right|^{p-2} T_{\varepsilon}^{+} \nabla u_{\varepsilon}^{+}\left(T_{\varepsilon}^{+} u^{\prime}, 0\right) d x d y_{1} d y_{2} \\
+\int_{R_{-}} \Pi_{\varepsilon}\left(\left|\nabla u_{\varepsilon}^{-}\right|^{p-2} \nabla u_{\varepsilon}^{-}\right)\left(\Pi_{\varepsilon} \nabla u_{\varepsilon}^{-}-\Pi_{\varepsilon}\left(u^{\prime}, 0\right)\right) d x d y \rightarrow 0
\end{gathered}
$$

since $T_{\varepsilon}\left(u_{\varepsilon}-u\right) \rightarrow 0$ strongly in $L^{p}\left((0,1) \times Y^{*}\right)$. Hence, as $\Pi_{\varepsilon} \nabla u_{\varepsilon}^{-}-\Pi_{\varepsilon}\left(u^{\prime}, 0\right) \rightarrow 0$ weakly in $L^{p}\left(R_{-}\right)$, we get from 49 and (50) that

$$
\lim _{\varepsilon \rightarrow 0} \int_{R_{-}}\left(\Pi_{\varepsilon}\left(\left|\nabla u_{\varepsilon}^{-}\right|^{p-2} \nabla u_{\varepsilon}^{-}\right)-\Pi_{\varepsilon}\left(\left|u^{\prime}\right|^{p-2}\left(u^{\prime}, 0\right)\right)\right)\left(\Pi_{\varepsilon} \nabla u_{\varepsilon}^{-}-\Pi_{\varepsilon}\left(u^{\prime}, 0\right)\right) d x d y=0 .
$$

On the other hand, we have

$$
\begin{aligned}
\int_{R_{-}}\left|\Pi_{\varepsilon} \nabla u_{\varepsilon}^{-}-\left(u^{\prime}, 0\right)\right|^{p} d x d y \leq & c \int_{R_{-}}\left|\Pi_{\varepsilon} \nabla u_{\varepsilon}^{-}-\Pi_{\varepsilon}\left(u^{\prime}, 0\right)\right|^{p} d x d y \\
& +c \int_{R_{-}}\left|\Pi_{\varepsilon}\left(u^{\prime}, 0\right)-\left(u^{\prime}, 0\right)\right|^{p} d x d y .
\end{aligned}
$$

If $p>2$, we get from (51) that

$$
\begin{aligned}
& \int_{R_{-}}\left|\Pi_{\varepsilon} \nabla u_{\varepsilon}^{-}-\Pi_{\varepsilon}\left(u^{\prime}, 0\right)\right|^{p} d x d y \leq \\
& \quad c \int_{R_{-}}\left(\Pi_{\varepsilon}\left(\left|\nabla u_{\varepsilon}^{-}\right|^{p-2} \nabla u_{\varepsilon}^{-}\right)-\Pi_{\varepsilon}\left(\left|u^{\prime}\right|^{p-2}\left(u^{\prime}, 0\right)\right)\right)\left(\Pi_{\varepsilon} \nabla u_{\varepsilon}^{-}-\Pi_{\varepsilon}\left(u^{\prime}, 0\right)\right) d x d y \\
& \quad \rightarrow 0 .
\end{aligned}
$$

For $1<p \leq 2$, using Hölder's inequality and (51), we obtain

$$
\begin{aligned}
& \int_{R_{-}}\left|\Pi_{\varepsilon} \nabla u_{\varepsilon}^{-}-\Pi_{\varepsilon}\left(u^{\prime}, 0\right)\right|^{p} d x d y \\
= & \int_{R_{-}} \frac{\left(\left|\Pi_{\varepsilon} \nabla u_{\varepsilon}^{-}\right|+\left|\Pi_{\varepsilon}\left(u^{\prime}, 0\right)\right|\right)^{\frac{(p-2) p}{2}}}{\left(\left|\Pi_{\varepsilon} \nabla u_{\varepsilon}^{-}\right|+\left|\Pi_{\varepsilon}\left(u^{\prime}, 0\right)\right|\right)^{\frac{(p-2) p}{2}}}\left|\Pi_{\varepsilon} \nabla u_{\varepsilon}^{-}-\Pi_{\varepsilon}\left(u^{\prime}, 0\right)\right|^{p} d x d y \\
\leq & \left(\int_{R_{-}}\left(\left|\Pi_{\varepsilon} \nabla u_{\varepsilon}^{-}\right|+\left|\Pi_{\varepsilon}\left(u^{\prime}, 0\right)\right|\right)^{(p-2)}\left|\Pi_{\varepsilon} \nabla u_{\varepsilon}^{-}-\Pi_{\varepsilon}\left(u^{\prime}, 0\right)\right|^{2} d x d y\right)^{p / 2} \\
& \left(\int_{R_{-}}\left(\left|\Pi_{\varepsilon} \nabla u_{\varepsilon}^{-}\right|+\left|\Pi_{\varepsilon}\left(u^{\prime}, 0\right)\right|\right)^{p} d x d y\right)^{(2-p) / 2}
\end{aligned}
$$




$$
\begin{aligned}
\leq & \left(c \int_{R_{-}}\left(\Pi_{\varepsilon}\left(\left|\nabla u_{\varepsilon}^{-}\right|^{p-2} \nabla u_{\varepsilon}^{-}\right)-\Pi_{\varepsilon}\left(\left|u^{\prime}\right|^{p-2}\left(u^{\prime}, 0\right)\right)\right)\left(\Pi_{\varepsilon} \nabla u_{\varepsilon}^{-}-\Pi_{\varepsilon}\left(u^{\prime}, 0\right)\right) d x d y\right)^{p / 2} \\
& \left(\int_{R_{-}}\left(\left|\Pi_{\varepsilon} \nabla u_{\varepsilon}^{-}\right|+\left|\Pi_{\varepsilon}\left(u^{\prime}, 0\right)\right|\right)^{p} d x d y\right)^{(2-p) / 2} \\
\rightarrow & 0 .
\end{aligned}
$$

Thus, as $\int_{R_{-}}\left|\Pi_{\mathcal{\varepsilon}}\left(u^{\prime}, 0\right)-\left(u^{\prime}, 0\right)\right|^{p} d x d y \rightarrow 0$, it follows that

$$
\Pi_{\varepsilon} \nabla u_{\varepsilon}^{-} \rightarrow\left(u^{\prime}, 0\right) \text { strongly in } L^{p}\left(R_{-}\right) \times L^{p}\left(R_{-}\right),
$$

and then,

$$
\left|\Pi_{\varepsilon} \nabla u_{\varepsilon}^{-}\right|^{p-2} \Pi_{\varepsilon} \nabla u_{\varepsilon}^{-} \rightarrow\left|u^{\prime}\right|^{p-2}\left(u^{\prime}, 0\right) \text { strongly in } L^{p^{\prime}}\left(R_{-}\right) \times L^{p^{\prime}}\left(R_{-}\right) .
$$

Finally, we can pass to the limit in $(48)$, for any test functions $\varphi \in W^{1, p}(0,1)$ to obtain

$$
\int_{R_{-}}\left|u^{\prime}\right|^{p-2} u^{\prime} \varphi^{\prime} d x d y+\frac{1}{L} \int_{(0,1) \times Y^{*}}|u|^{p-2} u \varphi d x d y_{1} d y_{2}=\int_{0}^{1} \hat{f} \varphi d x
$$

which is

$$
\int_{0}^{1} g_{0}\left|u^{\prime}\right|^{p-2} u^{\prime} \varphi^{\prime}+\frac{\left|Y^{*}\right|}{L}|u|^{p-2} u \varphi d x=\int_{0}^{1} \hat{f} \varphi d x
$$

for all $\varphi \in W^{1, p}(0,1)$, concluding the proof.

Corollary 5.3.1. Under hypothesis of Theorem 5.3, we have that the solutions of problem (2) satisfy

$$
\begin{aligned}
& \Pi_{\varepsilon} u_{\varepsilon}^{-} \rightarrow u \text { strongly in } W^{1, p}\left(R_{-}\right) \\
& \Pi_{\varepsilon}\left(\left|\nabla u_{\varepsilon}^{-}\right|^{p-2} \partial_{x} u_{\varepsilon}^{-}\right) \rightarrow\left|u^{\prime}\right|^{p-2} u^{\prime} \text { strongly in } L^{p}\left(R_{-}\right) \\
& \Pi_{\varepsilon}\left(\left|\nabla u_{\varepsilon}^{-}\right|^{p-2} \partial_{y} u_{\varepsilon}^{-}\right) \rightarrow 0 \text { strongly in } L^{p}\left(R_{-}\right) \\
& T_{\varepsilon}^{+}\left(\left|\nabla u_{\varepsilon}^{+}\right|^{p-2} \partial_{x} u_{\varepsilon}^{+}\right) \rightarrow 0 \text { strongly in } L^{p}\left((0,1) \times Y_{+}^{*}\right) \\
& T_{\varepsilon}^{+}\left(\left|\nabla u_{\varepsilon}^{+}\right|^{p-2} \partial_{y} u_{\varepsilon}^{+}\right) \rightarrow 0 \text { strongly in } L^{p}\left((0,1) \times Y_{+}^{*}\right)
\end{aligned}
$$

Moreover,

$$
\left\|\mid \nabla u_{\varepsilon}\right\|_{L^{p}\left(R_{+}^{\varepsilon}\right)} \rightarrow 0 .
$$

Proof. The convergence of (52) - (54) are proved in Theorem 5.3. For the convergence (55) and (56), we take as test function $\left(u_{\varepsilon}-u\right)$ in (48) to get from (52) - (54) and 47) that

$$
\int_{(0,1) \times Y_{+}^{*}} T_{\varepsilon}^{+}\left(\left|\nabla u_{\varepsilon}^{+}\right|^{p-2} \nabla u_{\varepsilon}^{+}\right) T_{\varepsilon}^{+}\left(\nabla u_{\varepsilon}-\nabla u\right) d x d y_{1} d y_{2} \rightarrow 0 .
$$

Thus

$$
\int_{(0,1) \times Y_{+}^{*}}\left|T_{\varepsilon}^{+} \nabla u_{\varepsilon}^{+}\right|^{p} \rightarrow 0
$$

which proves (55) and (56).

The last convergence follows from the above convergences and [6, Proposition 2.17].

Acknowledgements. The first author (JMA) is partially supported by grants MTM2016-75465-P, ICMAT Severo Ochoa project SEV-2015-0554, MINECO, Spain and Grupo de Investigación CADEDIF, UCM. The second author (JCN) is partially supported by CNPq (Brazil), and the third author (MCP) by CNPq 302960/2014-7 and FAPESP 2017/02630-2 (Brazil). 


\section{References}

[1] M. Anguiano and F. J. Z. Suárez-Grau. Homogenization of an incompressible non-Newtonian flow through a thin porous medium, Z. Angew. Math. Phys. (2017) DOI:10.1007/s00033-017-0790-Z

[2] J. M. Arrieta, A. N. Carvalho, M. C. Pereira, and R. P. Silva. Semilinear parabolic problems in thin domains with a highly oscillatory boundary. Nonlinear Analysis: Theory, Methods \& Applications, 74-15 (2011) 5111-5132.

[3] J. M. Arrieta and M. C. Pereira. Homogenization in a thin domain with an oscillatory boundary, J. Math. Pures et Appl. 96 (2011) 29-57.

[4] J.M. Arrieta, M.C. Pereira, The Neumann problem in thin domains with very highly oscillatory boundaries, J. Math. Anal. Appl. 444 (1) (2013) 86-104.

[5] J. M. Arrieta and M. Villanueva-Pesqueira, Unfolding operator method for thin domains with a locally periodic highly oscillatory boundary. SIAM J. of Math. Analysis 48-3 (2016) 1634-1671.

[6] J. M. Arrieta and M. Villanueva-Pesqueira. Thin domains with non-smooth oscillatory boundaries, J. of Math. Analysis and Appl. 446-1 (2017) 130-164.

[7] P. Bella, E. Feireisl and A. Novotny, Dimension reduction for compressible viscous fluids. Acta Appl. Math. 134 (2014) 111-121.

[8] M. Benes and I. Pazanin, Effective flow of incompressible micropolar fluid through a system of thin pipes. Acta Applicandae Mathematicae 143 (1) (2016) 29-43.

[9] G. Dal Maso and A. Defranceschi, Correctors for the homogenization of monotone operators. Diff. and Integral Eq. vol. 3, no. 3, (1990) pp. 1151-1166.

[10] P. Donato and G. Moscariello, "On the homogenization of some nonlinear problems in perforated domains," Rendiconti del Seminario Matematico della Università di Padova, vol. 84, pp. 91-108, 1990.

[11] A. Gaudiello, K. Hamdache, The polarization in a ferroelectric thin film: local and nonlocal limit problems. ESAIM Control Optim. Calc. Var. 19 (2013) 657-667.

[12] A. Gaudiello, K. Hamdache, A reduced model for the polarization in a ferroelectric thin wire. NoDEA Nonlinear Differential Equations Appl. 22 (6) (2015) 1883-1896.

[13] J. K. Hale and G. Raugel, Reaction-diffusion equations on thin domains. J. Math. Pures et Appl. 9 (71) (1992) 33-95.

[14] P. Lindqvist. Notes on the p-Laplace equation. University of Jyväskylä, 2017.

[15] T. A. Mel'nyk and A. V. Popov. Asymptotic analysis of boundary-value problems in thin perforated domains with rapidly varying thickness, Nonlinear Oscil. 13 (2010) 57-84.

[16] J. C. Nakasato and M. C. Pereira. The p-laplacian operator in oscillating thin domains. submitted, 2017.

[17] I. Pazanin, F.J. Súarez-Grau. Effects of rough boundary on the heat transfer in a thin-film flow, C. R. Mec. 341 (8) (2012) 646-652.

[18] M. C. Pereira. Asymptotic analysis of a semilinear elliptic equation in highly oscillating thin domains, Zeitschrift fur Angewandte Mathematik und Physik, 67 (2016) 1-14.

[19] M. C. Pereira and R. P. Silva. Remarks on the p-laplacian on thin domains, Progress in Nonlinear Diff. Eq. and Their Appl.(2015) 389-403.

[20] M.Villanueva-Pesqueira, Homogenization of Elliptic problems in thin domains with oscillatory boundaries, Ph.D. Thesis, Universidad Complutense de Madrid, 2016. 